\title{
Study of long-range orders of hard-core bosons coupled to cooperative normal modes in two-dimensional lattices
}

\author{
A. Ghosh and S. Yarlagadda \\ CMP Div., Saha Institute of Nuclear Physics, HBNI, Kolkata, India
}

\begin{abstract}
Understanding the microscopic mechanism of coexisting long-range orders (such as lattice supersolidity) in strongly correlated systems is a subject of immense interest. We study the possible manifestations of long-range orders, including lattice-supersolid phases with differently broken symmetry, in a two-dimensional square lattice system of hard-core bosons (HCBs) coupled to archetypal cooperative/coherent normal-mode distortions such as those in perovskites. At strong HCB-phonon coupling, using a duality transformation to map the strong-coupling problem to a weak-coupling one, we obtain an effective Hamiltonian involving nearest-neighbor, next-nearest-neighbor, and next-tonext-nearest-neighbor hoppings and repulsions. Using stochastic series expansion quantum Monte Carlo, we construct the phase diagram of the system. As coupling strength is increased, we find that the system undergoes a first-order quantum phase transition from a superfluid to a checkerboard solid at half filling and from a superfluid to a diagonal striped solid [with crystalline ordering wavevector $\vec{Q}=(2 \pi / 3,2 \pi / 3)$ or $(2 \pi / 3,4 \pi / 3)]$ at one-third filling without showing any evidence of supersolidity. On tuning the system away from these commensurate fillings, checkerboard supersolid is generated near half filling whereas a rare diagonal striped supersolid is realized near one-third filling. Interestingly, there is an asymmetry in the extent of supersolidity about one-third filling. Within our framework, we also provide an explanation for the observed checkerboard and stripe formations in $\mathrm{La}_{2-x} \mathrm{Sr}_{x} \mathrm{NiO}_{4}$ at $x=1 / 2$ and $x=1 / 3$.
\end{abstract}

\section{INTRODUCTION}

The origin and character of lattice supersolidity $\underline{1}$ [i.e., the single-phase coexistence of superconductivity/superfluidity and charge density wave (CDW) realized in discrete lattices] in naturally formed and artificially designed systems is a central issue in condensed matter physics. While phenomenological pictures 2,3 exist to explain lattice-supersolidity, a microscopic theory that elucidates the homogeneous coexistence is yet to be formulated. Supersolidity is observed in a variety of lattice systems such as the three-dimensional doped $\mathrm{BaBiO}_{3}{ }^{3,4}$; the layered dichalcogenides ${ }^{\underline{5}}$ and molecular crystals ${ }^{6}$; and the quasi-one-dimensional doped trichalcogenide $\mathrm{NbSe}_{3}{ }^{7}$ and doped spin ladder $\mathrm{Sr}_{14} \mathrm{Cu}_{24} \mathrm{O}_{41}{ }^{8-9}$. . Of importance are the class of materials that display superconductivity and diagonal long-range order due to strong electronphonon interaction such as $\mathrm{K}$ or $\mathrm{Pb}$ doped $\mathrm{BaBiO}_{3}$ (where a $10 \%$ change in the $\mathrm{Bi}-\mathrm{O}$ bond length $\frac{10}{10}$ has been observed) and the alkali metal fullerides 11 . Interestingly, $\mathrm{BaBiO}_{3}$ assumes perovskite structure with two adjacent oxygen octahedra sharing an oxygen leading to a cooperative breathing mode (CBM). Furthermore, $\mathrm{BaBiO}_{3}$ displays valence disproportionation with local cooper pairs [i.e., hard-core bosons (HCBs)] being formed and these HCBs couple to the $\mathrm{CBM}^{12}$.

As regards artificially engineered systems, cold bosonic atoms in optical lattices provide a fertile playground for actualizing exotic phases such as lattice-supersolid phases with differently broken symmetry. In fact, only recently supersolidity was experimentally produced in an optical lattice by generating effective long-range interactions

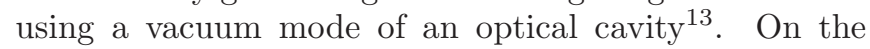
theoretical side, lattice supersolidity has been realized in two-dimensional (2D) square ${ }^{14-22}$, triangular ${ }^{23-29}$ and honeycomb $\underline{30,31}$ lattices as well as in a one-dimensional lattice $\underline{32} \underline{-34}$. By using extended boson Hubbard models involving hard-core bosons, while a supersolid has been been produced at a commensurate filling (i.e., half filling) in frustrated systems such as triangular lattices, commensurate supersolid has been unobtainable in unfrustrated systems such as square lattices. On the other hand, supersolids can be realized in square lattices at incommensurate fillings by a mechanism where particles (i.e., interstitials) or holes (i.e., vacancies) doped into a perfect crystal form a condensate by delocalizing in the crystalline order. Furthermore, although striped supersolidity has been achieved in Refs. 16 and 18 on square lattices, it is nondiagonal and characterized by density ordering wavevector $(\pi, 0)$ or $(0, \pi)$. Even though diagonal stripes [characterized by crystalline ordering wavevector $(2 \pi / 3,2 \pi / 3)$ or $(2 \pi / 3,4 \pi / 3)]$ have been observed in systems such as $\mathrm{La}_{2-x} \mathrm{Sr}_{x} \mathrm{NiO}_{4}$ (LSNO) at $x=1 / 3$ hole doping $35-41$ and predicted theoretically for long-range interactions in a lattice gas model at one-third filling $\stackrel{42}{ }$, so far the corresponding diagonal striped supersolid (dsSS) has been elusive on a square lattice (that is not subject to an external potential). Additionally, whether a cooperative electron-phonon interaction (that involves cooperative Jahn-Teller distortions) can explain the observed stripe charge order in LSNO is a controversial issue $\underline{\underline{43}} \underline{\underline{45}}$.

In the class of extended boson Hubbard models of the type $t_{1}-t_{2}-\ldots-t_{m}-V_{1}-V_{2}-\ldots-V_{n}$ [involving hoppings $t_{1}, t_{2}, t_{3}$, etc. and interactions $V_{1}, V_{2}, V_{3}$, etc. of ranges nearest neighbor (NN), next-nearest neighbor (NNN), next-to-next-nearest neighbor (NNNN), etc.] on a square lattice, the minimum model for realizing a checkerboard supersolid (cSS) is the $t_{2}-V_{1}$ mode 16.47 . It has also been shown that star/stripe supersolid [corresponding to crystalline ordering wavevector $(\pi, 0)$ or 
$(0, \pi)]$ can be realized in a $t_{1}-V_{1}-V_{2}$ model; at onefourth filling, a star solid results which is asymmetric with respect to doping with interstitials and vacancies 16 . Identifying the relevant extended boson Hubbard model for obtaining the dsSS around one-third filling and characterizing the state are still open problems.

Here, inspired by the doped bismuthate systems, we develop a microscopic theory of HCBs strongly coupled to the cooperative breathing mode in a $2 \mathrm{D}$ perovskite lattice. The effective Hamiltonian for the HCBs is shown to be an extended boson Hubbard model of the form $t_{1}-t_{2}-t_{3}-V_{1}-V_{2}-V_{3}$. The $V_{1}, V_{2}$, and $V_{3}$ repulsive interactions correspond to the minimum interactions needed to realize the diagonal stripedorder at one-third filling. Unlike many lattice models of the extended boson Hubbard type, the parameters (i.e., hopping term, strength of HCB-phonon coupling, and phonon frequency) in our $t_{1}-t_{2}-t_{3}-V_{1}-V_{2}-V_{3}$ model either can be determined from band-structure calculations or can be obtained from experiments. Supersolidity in our model results only away from one-third filling and is shown to be asymmetric with respect to doping the commensurate diagonal-striped solid (dsS) with vacancies and interstitials. Although checkerboard supersolidity (away from half-filling) and diagonal striped supersolidity (away from one-third filling) are realized, there is no direct supersolid-supersolid phase transition between the two phases. We also show that our cooperative HCB-phonon framework can be extended to study charge order in LSNO; we demonstrate that the observed diagonal-stripe order at one-third filling and the checkerboard order at half filling in LSNO can be explained by invoking cooperative Jahn-Teller effect.

The paper is organized as follows. In Sec. II] we derive an effective Hamiltonian of the system using a nonperturbative treatment. Next, in Sec. III we briefly describe the numerical procedure, as well as the quantities/parameters used in our study. Then, we discuss the results in Sec. IV] followed by a comparison with experimental observations in Sec. V] Finally, in Sec. VI, we conclude.

\section{EFFECTIVE HAMILTONIAN}

We start with a 2D model of HCBs depicted in Fig. 1. The HCBs interact with the in-plane (xy) oxygen atoms via CBM, whereas the nature of the interaction is noncooperative in the case of the out-of-plane oxygen atoms in the z-direction. The Hamiltonian of such a system can be written as $H=H_{t}+H_{I}+H_{l}$, where the hopping term $H_{t}$ is given by

$$
H_{t}=-t \sum_{i, j}\left(d_{i+1, j}^{\dagger} d_{i, j}+d_{i, j+1}^{\dagger} d_{i, j}+\text { H.c. }\right),
$$

with $d_{i, j}\left(d_{i, j}^{\dagger}\right)$ being the destruction (creation) operator of a HCB at the hopping site $(i, j)$ and $t$ being the hopping integral. The second term $H_{I}$ in the Hamiltonian,

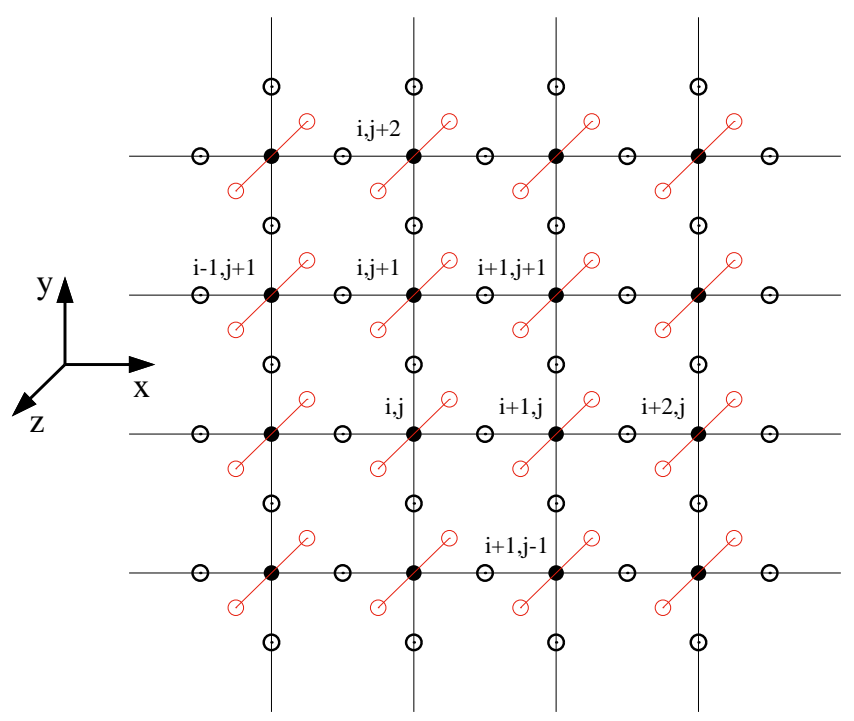

FIG. 1. (Color online) Two-dimensional cooperative breathing mode (CBM) system with hopping sites of hard-corebosons (filled circles), in-plane oxygen atoms (black empty circles) and out-of-plane oxygen atoms (red empty circle). Only the in-plane oxygens are involved in cooperative distortions.

which represents the HCB-phonon interaction, has the form

$$
\begin{aligned}
& H_{I}=-g \omega_{0} \sum_{i, j}\left[\left(a_{x ; i, j}^{\dagger}+a_{x ; i, j}\right)\left(n_{i, j}-n_{i+1, j}\right)\right. \\
& \left.+\left(b_{y ; i, j}^{\dagger}+b_{y ; i, j}\right)\left(n_{i, j}-n_{i, j+1}\right)+\gamma\left(c_{z ; i, j}^{\dagger}+c_{z ; i, j}\right) n_{i, j}\right],
\end{aligned}
$$

where $\gamma=\sqrt{2}, g$ is the HCB-phonon coupling constant, and $\omega_{0}$ is the optical-phonon frequency. The terms $\left(a_{x ; i, j}^{\dagger}+a_{x ; i, j}\right) / \sqrt{2 M \omega_{0}}$ and $\left(b_{y ; i, j}^{\dagger}+b_{y ; i, j}\right) / \sqrt{2 M \omega_{0}}$ denote the displacement of the oxygen atom that is next to the $(i, j)$-th hopping site and in the positive $\mathrm{x}$ - and $\mathrm{y}$-directions, respectively; here, $M$ is the mass of oxygen atom. The relative displacement of the two out-of-plane oxygens next to the $(i, j)$-th site couples to the HCB at $(i, j)$-th site and is denoted by $\left(c_{z ; i, j}^{\dagger}+c_{z ; i, j}\right) / \sqrt{2 \frac{M}{2} \omega_{0}}$ with $M / 2$ being the reduced mass of the oxygen pairs. The expressions $\left(n_{i, j}-n_{i+1, j}\right)$ and $\left(n_{i, j}-n_{i, j+1}\right)$ in the first and second terms of Eq. (2) take care of the cooperative HCB-phonon interaction along the $\mathrm{x}$ - and $\mathrm{y}$ directions, respectively. In the third term, note that we have only $n_{i, j}$ because of the non-cooperative nature of the HCB-phonon interaction along the z-direction. Furthermore, the last term in the Hamiltonian (i.e., the lattice term $H_{l}$ ), representing simple harmonic oscillators, is of the form

$$
H_{l}=\omega_{0} \sum_{i, j}\left(a_{x ; i, j}^{\dagger} a_{x ; i, j}+b_{y ; i, j}^{\dagger} b_{y ; i, j}+\eta c_{z ; i, j}^{\dagger} c_{z ; i, j}\right),
$$

with $\eta=1$. 
We consider systems in the non-adiabatic regime $\left(t / \omega_{0} \leqslant 1\right)$ and strong-coupling region (large $\left.g^{2}\right)$. To produce an effective polaronic Hamiltonian, we employ a duality transformation where the strong-coupling problem in the original frame of reference [with small parameter $\left.\propto\left(g \omega_{0}\right) / t\right]$ is transformed into a weak-coupling problem in a dual frame of reference [with small parameter $\propto t /\left(g \omega_{0}\right)$, i.e., inverse of the small parameter in the original frame of reference]. To achieve the above end, we need to modify the Lang-Firsov transformation $\underline{\underline{48}}$ so as to take into account the cooperative nature of the distortions along the $\mathrm{x}$ - and $\mathrm{y}$-directions and non-cooperative nature in the z-direction. This involves the following canonical transformation $\tilde{H}=\exp (S) H \exp (-S)$ where $S$ is given by

$$
\begin{aligned}
S & =-g \sum_{i, j}\left[\left(a_{x ; i, j}^{\dagger}-a_{x ; i, j}\right)\left(n_{i, j}-n_{i+1, j}\right)\right. \\
& \left.+\left(b_{y ; i, j}^{\dagger}-b_{y ; i, j}\right)\left(n_{i, j}-n_{i, j+1}\right)+\gamma\left(c_{z ; i, j}^{\dagger}-c_{z ; i, j}\right) n_{i, j}\right] .
\end{aligned}
$$

The transformed Hamiltonian can be written as $\tilde{H}=$ $H_{0}+H_{1}$, where the unperturbed Hamiltonian is given by

$$
\begin{aligned}
H_{0} & =\omega_{0} \sum_{i, j}\left(a_{x ; i, j}^{\dagger} a_{x ; i, j}+b_{y ; i, j}^{\dagger} b_{y ; i, j}+\eta c_{z ; i, j}^{\dagger} c_{z ; i, j}\right) \\
& -E_{p} \sum_{i, j} n_{i, j}+2 V_{p} \sum_{i, j}\left(n_{i, j} n_{i+1, j}+n_{i, j} n_{i, j+1}\right) \\
& -t e^{-\left(E_{p}+V_{p}\right) / \omega_{0}} \sum_{i, j}\left(d_{i+1, j}^{\dagger} d_{i, j}+d_{i, j+1}^{\dagger} d_{i, j}+\text { H.c. }\right),
\end{aligned}
$$

and the perturbation by

$$
\begin{aligned}
H_{1}= & \sum_{i, j} H_{1 i, j} \\
=-t e^{-\left(E_{p}+V_{p}\right) / \omega_{0}} & \sum_{i, j}\left[d_{i+1, j}^{\dagger} d_{i, j}\left(\tau_{+x}^{i j}{ }^{\dagger} \tau_{-x}^{i j}-1\right)\right. \\
& \left.+d_{i, j+1}^{\dagger} d_{i, j}\left(\tau_{+y}^{i j}{ }^{\dagger} \tau_{-y}^{i j}-1\right)+\text { H.c. }\right],
\end{aligned}
$$

where

$$
\begin{aligned}
\tau_{ \pm x}^{i j} & =\exp \left[ \pm g\left(2 a_{i, j}-a_{i-1, j}-a_{i+1, j}\right)\right. \\
& \left. \pm g\left(b_{i+1, j-1}+b_{i, j}-b_{i, j-1}-b_{i+1, j}\right) \pm \gamma g\left(c_{i, j}-c_{i+1, j}\right)\right]
\end{aligned}
$$

and

$$
\begin{aligned}
\tau_{ \pm y}^{i j} & =\exp \left[ \pm g\left(2 b_{i, j}-b_{i, j-1}-b_{i, j+1}\right)\right. \\
& \left. \pm g\left(a_{i-1, j+1}+a_{i, j}-a_{i-1, j}-a_{i, j+1}\right) \pm \gamma g\left(c_{i, j}-c_{i, j+1}\right)\right]
\end{aligned}
$$

Here $E_{p}=\left(4+\gamma^{2}\right) g^{2} \omega_{0}$ is the polaronic energy and $2 V_{p}=$ $2 g^{2} \omega_{0}$ represents the nearest-neighbor repulsion for the HCBs.
The eigenstates of the unperturbed Hamiltonian $H_{0}$, relevant for perturbation theory are $|n, m\rangle=|n\rangle_{h c b} \otimes$ $|m\rangle_{p h}$, with $|0,0\rangle$ being the ground state with no phonons. The corresponding eigenenergies of such states are given by $E_{n, m}=E_{n}^{h c b}+E_{m}^{p h}$. Similar to the case of one-dimensional $\mathrm{CBM}$ mode $\underline{49}^{49}$, we also have $\left\langle n, 0\left|H_{1}\right| n, 0\right\rangle=0$, which yields the first-order perturbation term $\left\langle 0,0\left|H_{1}\right| 0,0\right\rangle=0$. In the region of interest in the parameter space, we note that $t e^{-\left(E_{p}+V_{p}\right) / \omega_{0}}<<\omega_{0}$; we perform second order perturbation theory similar to that in the $1 \mathrm{D}$ CBM mode $\underline{49}$ and obtain the effective Hamiltonian to be

$$
H_{\text {eff }}=\left\langle\left. 0\right|_{p h} H_{0} \mid 0\right\rangle_{p h}+H^{(2)},
$$

where

$$
H^{(2)}=\sum_{i, j, k, l} \sum_{m} \frac{\left\langle\left. 0\right|_{p h} H_{1 i, j} \mid m\right\rangle_{p h}\left\langle\left. m\right|_{p h} H_{1 k, l} \mid 0\right\rangle_{p h}}{E_{0}^{p h}-E_{m}^{p h}} .
$$

One can easily see that the first term in $H_{\text {eff }}$ is

$$
\begin{aligned}
& \left\langle\left. 0\right|_{p h} H_{0} \mid 0\right\rangle_{p h}=-E_{p} \sum_{i, j} n_{i, j} \\
& +2 V_{p} \sum_{i, j}\left(n_{i, j} n_{i+1, j}+n_{i, j} n_{i, j+1}\right) \\
& -t e^{-\left(E_{p}+V_{p}\right) / \omega_{0}} \sum_{i, j}\left(d_{i+1, j}^{\dagger} d_{i, j}+d_{i, j+1}^{\dagger} d_{i, j}+\text { H.c. }\right),
\end{aligned}
$$

whereas the simplification of the second term (i.e, $H^{(2)}$ ) requires quite a bit of algebra. We extend the derivation of the effective Hamiltonian for the 1D CBM case $e^{49}$ to our 2D case as well. As shown by using Schrieffer-Wolff transformation in Appendix A of Refs. 50 and 51, since $t e^{-\left(E_{p}+V_{p}\right) / \omega_{0}}<<\omega_{0}, H_{\text {eff }}$ represents the exact Hamiltonian up to second order in perturbation. The small parameter here is given by $\left[\frac{t^{2}}{2\left(E_{v}+V_{p}\right) \omega_{0}}\right]^{\frac{1}{2}}$ whose derivation is similar to that in Ref. 52. For the second term $H^{(2)}$ in $H_{\text {eff }}$, we obtain the terms given in the following subsections.

\section{A. Nearest-neighbor (NN) repulsion}

The NN repulsion term comes from a process where a particle jumps to a neighboring site and comes back. In 2D, this term further consists of two parts: $\sum_{i, j}\left[n_{i, j}\left(1-n_{i+1, j}\right)+n_{i+1, j}\left(1-n_{i, j}\right)\right]$ and $\sum_{i, j}\left[n_{i, j}\left(1-n_{i, j+1}\right)+n_{i, j+1}\left(1-n_{i, j}\right)\right]$. Following a procedure explained in Appendix A, we get the expression for this process to be

$$
-V_{z} \sum_{i, j}\left[n_{i, j}\left(1-n_{i+1, j}\right)+n_{i, j}\left(1-n_{i, j+1}\right)\right],
$$


with $V_{z} \approx \frac{2 t^{2}}{2 E_{p}+2 V_{p}}$. The denominator $2 E_{p}+2 V_{p}$ in $V_{z}$ is the difference of the energy of the intermediate state (i.e., $E_{p}+2 V_{p}$ corresponding to the particle in the intermediate site) and the energy of the initial state $\left(-E_{p}\right)$. The exact expression for $V_{z}$ is derived in Appendix A.

\section{B. Next-nearest-neighbor (NNN) and} next-to-next-nearest-neighbor (NNNN) repulsions

We first make an important point while considering a process of a particle hopping to a neighboring site and coming back. In 2D, excluding the originating site, we must take into account the occupancy information about all the three remaining NN sites of the intermediate site of the hopping process. For example, consider a process where a HCB at site $(i, j)$ hops to its neighboring site $(i+1, j)$ and comes back. For this process, we need to keep in mind the occupancy of the sites $(i+2, j)$, $(i+1, j+1)$ and $(i+1, j-1)$, which are the three relevant neighboring sites of the intermediate site $(i+1, j)$ (see Fig. 1). Depending on whether these sites are occupied or empty, the coefficient of the process will be modified accordingly. Essentially there are four cases: 1) all the three NN sites are empty ; 2) any one of the three neighboring sites is occupied ; 3) any two of the NN sites are occupied; and 4) all the three neighboring sites are occupied. Considering all the cases above, we end up with the following NNN and NNNN repulsion terms in $H^{(2)}$ as detailed in Appendix B.

\section{NNN repulsion along diagonals}

The first term is the NNN repulsion which acts along the diagonals of the square lattice; it is given by

$$
V_{2} \sum_{i, j}\left(n_{i, j} n_{i+1, j+1}+n_{i, j} n_{i-1, j+1}\right),
$$

where

$$
\begin{aligned}
V_{2}= & 2 t^{2}\left[\left(\frac{1}{2}-m\right)^{2} \frac{2 V_{p}}{\left(E_{p}+V_{p}\right)\left(E_{p}+2 V_{p}\right)}\right. \\
& +\left(\frac{1}{4}-m^{2}\right) \frac{4 E_{p} V_{p}}{\left(E_{p}+V_{p}\right)\left(E_{p}+2 V_{p}\right)\left(E_{p}+3 V_{p}\right)} \\
& \left.+\left(\frac{1}{2}+m\right)^{2} \frac{2 E_{p} V_{p}}{\left(E_{p}+2 V_{p}\right)\left(E_{p}+3 V_{p}\right)\left(E_{p}+4 V_{p}\right)}\right],
\end{aligned}
$$

with $m$ being the magnetization of the system.

\section{NNNN repulsion along the $x$ - and $y$-axes}

We find the second term to be the NNNN repulsion which acts along the $\mathrm{x}$ - and $\mathrm{y}$-axes of the square lattice; it is given by

$$
V_{3} \sum_{i, j}\left(n_{i, j} n_{i+2, j}+n_{i, j} n_{i, j+2}\right)
$$

with $V_{3}=\frac{V_{2}}{2}$.

It is important to note that, in the absence of the $\mathrm{NN}$ repulsion $2 V_{p}$, we obtain expressions for $V_{z}, V_{2}$, and $V_{3}$ consistent with the non-cooperative treatment of the electron-phonon interaction in Ref. 21.

\section{NNN and NNNN hoppings}

The remaining terms in $H^{(2)}$ are the hoppings of the HCBs to the NNN and NNNN sites. Similar to the NNN and NNNN repulsions, the hopping contributions of the HCBs can also be divided into two types: NNN hopping along the diagonals and NNNN hopping along the $\mathrm{x}$ - and y-axes (see Appendix C for details).

\section{NNN hopping along diagonals}

While calculating the coefficient of the NNN hopping, we have to keep in mind the fact that the HCB passes through an intermediate site while hopping to its NNN site. So the coefficient must depend on the occupancy of the two neighboring sites of the intermediate site. For example, if a $\mathrm{HCB}$ at site $(i, j)$ is hopping to its rightupper diagonal site, i.e., $(i+1, j+1)$, it can follow any one of the two possible paths: a) first going along $\mathrm{x}$-axis to the $(i+1, j)$-th site and then along $\mathrm{y}$-axis to the $(i+1, j+1)$-th site; and b) the interchanged process, i.e., hopping along the $\mathrm{y}$-axis first to the $(i, j+1)$-th site followed by a hop along the $\mathrm{x}$-axis to the $(i+1, j+1)$-th site (see Fig. 10). For the first path, the coefficient of the hopping depends on whether the two sites $(i+2, j)$ and $(i+1, j-1)$, which are $\mathrm{NN}$ of the intermediate site $(i+1, j)$, are occupied or empty. On the other hand, for the second path, the hopping coefficient depends on the occupancy of the two neighboring sites of the intermediate site $(i, j+1)$, i.e., $(i-1, j+1)$ and $(i, j+2)$. To calculate the NNN hopping coefficient, first we forget about the occupancy of the two neighbors of the intermediate site; then, the NNN hopping along the diagonals is obtained to be

$$
-\frac{2 t^{2} e^{-E_{p} / \omega_{0}}}{E_{p}+2 V_{p}} \sum_{i, j}\left(d_{i+1, j+1}^{\dagger} d_{i, j}+d_{i-1, j+1}^{\dagger} d_{i, j}+\text { H.c. }\right) \text {, }
$$

where the coefficient $\frac{2 t^{2} e^{-E_{p} / \omega_{0}}}{E_{p}+2 V_{p}}$ is an approximation with the exact expression being given in Appendix C.

Now, taking the two neighbors of the intermediate site into account, the NNN hopping term along the diagonals of the square lattice gets modified to be

$$
-t_{2} \sum_{i, j}\left(d_{i+1, j+1}^{\dagger} d_{i, j}+d_{i-1, j+1}^{\dagger} d_{i, j}+\text { H.c. }\right),
$$


where

$$
\begin{aligned}
t_{2}=\frac{2 t^{2} e^{-E_{p} / \omega_{0}}}{E_{p}+2 V_{p}}[ & \left(\frac{1}{2}-m\right)^{2}+\left(\frac{1}{4}-m^{2}\right) \frac{2 E_{p}+4 V_{p}}{E_{p}+4 V_{p}} \\
& \left.+\left(\frac{1}{2}+m\right)^{2} \frac{E_{p}+2 V_{p}}{E_{p}+6 V_{p}}\right]
\end{aligned}
$$

\section{NNNN hopping along the $x$ - and $y$-axes}

Next, we consider the hopping of the HCBs to the NNNN sites along the $\mathrm{x}$ - and $\mathrm{y}$-axes of the square lattice. Similar to the previous case, the coefficient of the hopping in this case, depends on the occupancy of the two neighboring sites of the intermediate site. For example, if a HCB is hopping from site $(i, j)$ to its NNNN site $(i+2, j)$, it has to pass through the intermediate site $(i+1, j)$ (see Fig. 11). So, the coefficient for this process depends on whether the neighboring sites of site $(i+1, j)$, i.e., $(i+1, j+1)$ and $(i+1, j-1)$, are occupied or empty. Taking into account all the occupancy possibilities of the neighboring sites of the intermediate site, we get the NNNN hopping term to be

$$
-t_{3} \sum_{i, j}\left(d_{i+2, j}^{\dagger} d_{i, j}+d_{i, j+2}^{\dagger} d_{i, j}+\text { H.c. }\right) \text {, }
$$

with $t_{3}=\frac{t_{2}}{2}$.

Again, it should be pointed out that, in the absence of the NN repulsion $2 V_{p}$, the expressions for $t_{2}$ and $t_{3}$ simplify to be consistent with the results of the noncooperative analysis of the electron-phonon interaction in Ref. 21.

Finally, taking all the terms present in $H^{(2)}$ into account, $H_{\text {eff }}$ in Eq. (7) reduces to

$$
\begin{aligned}
H_{\text {eff }}= & -\left(E_{p}+2 V_{z}\right) \sum_{i, j} n_{i, j} \\
& -t_{1} \sum_{i, j}\left(d_{i+1, j}^{\dagger} d_{i, j}+d_{i, j+1}^{\dagger} d_{i, j}+\text { H.c. }\right) \\
& +V_{1} \sum_{i, j}\left(n_{i, j} n_{i+1, j}+n_{i, j} n_{i, j+1}\right) \\
& -t_{2} \sum_{i, j}\left(d_{i+1, j+1}^{\dagger} d_{i, j}+d_{i-1, j+1}^{\dagger} d_{i, j}+\text { H.c. }\right) \\
& +V_{2} \sum_{i, j}\left(n_{i, j} n_{i+1, j+1}+n_{i, j} n_{i-1, j+1}\right) \\
& -t_{3} \sum_{i, j}\left(d_{i+2, j}^{\dagger} d_{i, j}+d_{i, j+2}^{\dagger} d_{i, j}+\text { H.c. }\right) \\
& +V_{3} \sum_{i, j}\left(n_{i, j} n_{i+2, j}+n_{i, j} n_{i, j+2}\right)
\end{aligned}
$$

where $t_{1}=t e^{-\left(E_{p}+V_{p}\right) / \omega_{0}}, \quad V_{1}=2 V_{p}+V_{z}$, and the expressions for all the remaining terms, $V_{z}, t_{2}, t_{3}, V_{2}$, and $V_{3}$, being the same as defined earlier.

\section{NUMERICAL CALCULATIONS}

To study the phase diagram of our effective Hamiltonian of HCBs, we use quantum Monte Carlo (QMC) simulation employing the stochastic-series-expansion (SSE) technique. The first step required for SSE is to rewrite the Hamiltonian in terms of spin- $1 / 2$ operators. Identifying the relations between the operators for HCBs and those for spin- $1 / 2$ particles as $d_{i, j}^{\dagger}=S_{i, j}^{+}, d_{i, j}=S_{i, j}^{-}$ and $n_{i, j}=S_{i, j}^{z}+\frac{1}{2}$, we recast our effective Hamiltonian for $\mathrm{HCBs}$, in units of $2 t_{1}$, as an extended XXZ spin- $1 / 2$ Hamiltonian, given by

$$
\begin{aligned}
& H=\sum_{i, j}[-\frac{1}{2}\left(S_{i+1, j}^{+} S_{i, j}^{-}+S_{i, j+1}^{+} S_{i, j}^{-}+\text {H.c. }\right) \\
&\left.+\Delta_{1}\left(S_{i, j}^{z} S_{i+1, j}^{z}+S_{i, j}^{z} S_{i, j+1}^{z}\right)\right] \\
&+\sum_{i, j}[-\frac{J_{2}}{2}\left(S_{i+1, j+1}^{+} S_{i, j}^{-}+S_{i-1, j+1}^{+} S_{i, j}^{-}+\text {H.c. }\right) \\
&\left.+\Delta_{2}\left(S_{i, j}^{z} S_{i+1, j+1}^{z}+S_{i, j}^{z} S_{i-1, j+1}^{z}\right)\right] \\
&+\sum_{i, j}\left[-\frac{J_{3}}{2}\left(S_{i+2, j}^{+} S_{i, j}^{-}+S_{i, j+2}^{+} S_{i, j}^{-}+\text {H.c. }\right)\right. \\
&\left.\quad+\Delta_{3}\left(S_{i, j}^{z} S_{i+2, j}^{z}+S_{i, j}^{z} S_{i, j+2}^{z}\right)\right] \\
&-h_{0} \sum_{i, j} S_{i, j}^{z} .
\end{aligned}
$$

Looking at Eqs. (18) and (19), one can easily see that $J_{2}=t_{2} / t_{1}, J_{3}=t_{3} / t_{1}, \Delta_{1}=V_{1} /\left(2 t_{1}\right), \Delta_{2}=V_{2} /\left(2 t_{1}\right)$, $\Delta_{3}=V_{3} /\left(2 t_{1}\right)$ and $h_{0}=E_{p}+2 V_{z}-2 V_{1}-2 V_{2}-2 V_{3}$; here, $J_{i}$ and $\Delta_{i}$ are the transverse and longitudinal couplings, respectively.

Now, to figure out the phase diagram of the system, we need to study the Hamiltonian at various filling-fractions of HCBs. To vary the number of HCBs in the system, or in other words to tune the magnetization of the spin$1 / 2$ system, we replace the constant $h_{0}$ by a variable $h$ in the term $-h_{0} \sum_{i, j} S_{i, j}^{z}$ of the Hamiltonian $H$ given by Eq. (19); here $h$ is taken as the external magnetic field in units of $2 t_{1}$. By tuning the external magnetic field $h$, we can actually tune the magnetization of the system and study the behavior of the system at various fillings.

We use two kinds of order parameter: structure factor $S(\vec{Q})$ (to identify diagonal long-range order) and superfluid density $\rho_{s}$ (to identify off-diagonal long-range order) and construct the phase diagram. The structure factor per site is defined as

$$
S(\vec{Q})=\frac{1}{N_{s}^{2}} \sum_{i, j} \sum_{m, n} e^{i \vec{Q} \cdot\left(\vec{R}_{i, j}-\vec{R}_{m, n}\right)}\left\langle S_{i, j}^{z} S_{m, n}^{z}\right\rangle,
$$

with $\langle\ldots\rangle$ being the ensemble average. We study $S(\vec{Q})$ at all values of $\vec{Q}$ and identify those that produce peaks in the structure factor. Here we would like to point out that the maximum possible value of $S(\vec{Q})$ is 0.25 . 


\begin{tabular}{|c|c|c|c|}
\hline$\tilde{g}$ & $\Delta_{1}$ & $\left(\Delta_{2}\right)_{\max }$ & $\left(J_{2}\right)_{\max }$ \\
\hline 1.0 & 1.7436 & 0.4757 & 1.6486 \\
\hline 1.5 & 5.7744 & 0.7379 & 0.8760 \\
\hline 1.8 & 16.6463 & 1.3791 & 0.7007 \\
\hline 2.0 & 39.2161 & 2.3887 & 0.6327 \\
\hline 2.25 & 131.8584 & 5.4612 & 0.5818 \\
\hline 2.5 & 507.9968 & 14.5044 & 0.5584 \\
\hline 3.0 & 10896.8217 & 157.5599 & 0.5744 \\
\hline
\end{tabular}

TABLE I. Values of NN longitudinal coupling $\Delta_{1}$ and maximum values of NNN longitudinal coupling $\Delta_{2}$ and NNN transverse coupling $J_{2}$ for different values of $\tilde{g}$.

The superfluid density is expressed in terms of the winding numbers, $W_{x}$ and $W_{y}$, in the x- and y-directions as 53

$$
\rho_{s}=\frac{1}{2 \beta}\left\langle W_{x}^{2}+W_{y}^{2}\right\rangle
$$

The winding number $W_{x}$ along the x-direction can be calculated as $W_{x}=\frac{1}{L_{x}}\left(N_{x}^{+}-N_{x}^{-}\right)$, where $N_{x}^{+}$and $N_{x}^{-}$ denote the total number of operators transporting spin in positive and negative x-directions, respectively and $L_{x}$ denotes the length of the lattice along the $\mathrm{x}$-direction.

We now discuss the values of different parameters in our Hamiltonian given by Eq. (19) and used in our numerical calculations. We concentrate on the case $t / \omega_{0}=1.0$ for the construction of our phase diagram. Since $\gamma=\sqrt{2}$, we set $\tilde{g}^{2}=7 g^{2}$ so as to get the simple expression $E_{p}+V_{p}=\tilde{g}^{2} \omega_{0}$. The coefficients $J_{2}\left(=2 J_{3}\right)$
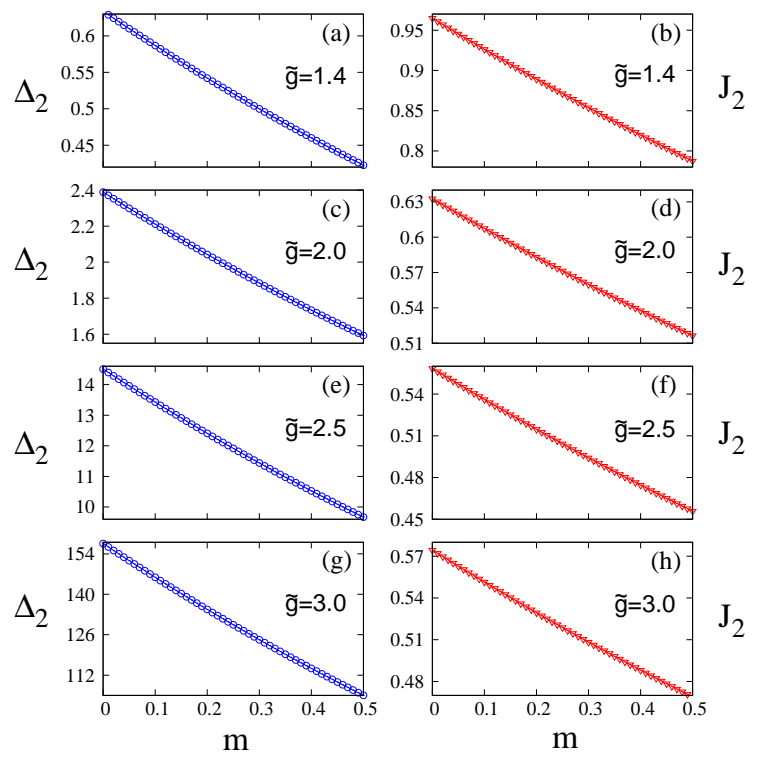

FIG. 2. (Color online) Dependence of NNN longitudinal coupling $\Delta_{2}$ and NNN transverse coupling $J_{2}$ on magnetization $m$ as derived from Eqs. (12), (16), (18), and (19) for the following cases: (a) \& (b) at $\tilde{g}=1.4$; (c) \& (d) at $\tilde{g}=2.0$; (e) $\&(\mathrm{f})$ at $\tilde{g}=2.5$; and (g) \& (h) at $\tilde{g}=3.0$. and $\Delta_{2}\left(=2 \Delta_{3}\right)$ depend on the magnetization $m$ of the system. While Fig. 2 depicts that $J_{2}$ and $\Delta_{2}$ values (at various couplings $\tilde{g}$ ) monotonically decreases with increasing magnetization $m$, Table \ shows the values of $\Delta_{1}$ and the maximum values of $\Delta_{2}$ and $J_{2}$ for different values of $\tilde{g}$. As one can see, $\Delta_{1} /\left(\Delta_{2}\right)_{\max }$ increases monotonically approximately from 3.665 to 69.159 as $\tilde{g}$ is varied from 1.0 to 3.0. At larger values of $\tilde{g}$, when $\Delta_{1}$ and $\Delta_{2}$ assume large values, our numerical calculations suffer from significant slowing down resembling the situation in Ref. 21; with our computational constraints we cannot use exact values when $\Delta_{1}$ and $\Delta_{2}$ assume large values. We can set a cut-off for the parameters $\Delta_{1}$ and $\Delta_{2}$ above which the essential physics for our system remains unaltered. Similar to Ref. 21, the upper cut-off for $\Delta_{1}$ is 16 . Furthermore, to identify the cut-off for $\Delta_{2}$, we need to find out the lowest value of $\Delta_{1} / \Delta_{2}$ which can be used without changing the essential physics. To this end, we have calculated the superfluid density and structure factor at half-filling (where $\Delta_{2}=\left(\Delta_{2}\right)_{\max }$ ) for the following set of values of $\left(\Delta_{1},\left(\Delta_{2}\right)_{\max }\right):(20,4),(20,5),(20,6)$, $(16,5),(20,7),(17,6),(16,7)$, and $(20,9)$ with the value of $\Delta_{1} /\left(\Delta_{2}\right)_{\max }$ being $5,4,3.33,3.2,2.86,2.83,2.29$, and 2.22 , respectively. Numerical results show that for the first four cases, where $\Delta_{1}>3\left(\Delta_{2}\right)_{\max }$, at half-filling the system manifests a checkerboard solid (cS) with a peak in the structure factor $S(\pi, \pi)$. On the other hand, for the last four cases where $2\left(\Delta_{2}\right)_{\max }<\Delta_{1}<3\left(\Delta_{2}\right)_{\max }$, at half-filling the system produces a completely different type of solid depicted in Fig. 3 (which we call honeycomb-like solid), indicated by a peak in $S(\pi / 2, \pi)$ or $S(\pi, \pi / 2)$. The reason can be explained as follows. In the cS phase each particle feels $6\left(\Delta_{2}\right)_{\max }$ amount repulsion, whereas in the honeycomb-like solid the repulsion felt by each particle is $\Delta_{1}+3\left(\Delta_{2}\right)_{\max }$. The checkerboard solid will be favored over the honeycomb-like solid only if $\Delta_{1}+3\left(\Delta_{2}\right)_{\max }>6\left(\Delta_{2}\right)_{\max }$, i.e., $\Delta_{1}>3\left(\Delta_{2}\right)_{\max }$. Therefore to capture the correct physics of our system, the minimum value of $\Delta_{1} / \Delta_{2}$ must be greater than 3. Keeping all these facts in mind, we set the cut-off values to be $\Delta_{1}=16$ and $\Delta_{2}=5$ (with $\Delta_{3}=\frac{\Delta_{2}}{2}$ ), so that the physics of the system still remains the same.

All numerical results in Figs. 410 have been obtained in a $18 \times 18$ lattice with $t / \omega_{0}=1.0$.

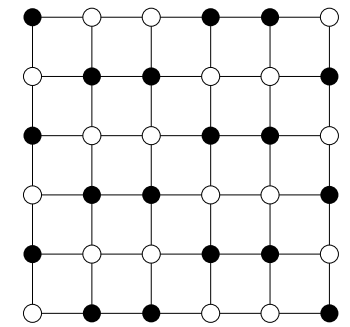

(a)

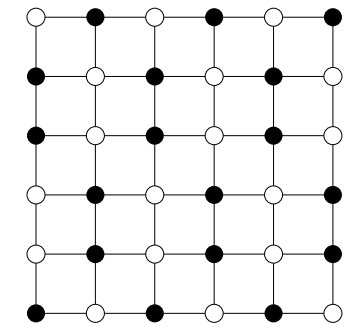

(b)
FIG. 3. Two types of honeycomb-like solid depicted by a peak in (a) $S(\pi / 2, \pi)$ and (b) $S(\pi, \pi / 2)$ 


\section{RESULTS AND DISCUSSIONS}

To determine the various phases of our $2 \mathrm{D} t_{1}-t_{2}-t_{3}-$ $V_{1}-V_{2}-V_{3}$ model, one needs to understand the interplay between different types of hopping and repulsion. To construct the phase diagram, we vary the magnetization $m$ from 0 to 0.5 ; this corresponds to varying the particle filling $\rho$ from $1 / 2$ to 1 . Due to particle-hole symmetry of the Hamiltonian, the physics at any filling-fraction for particles is identical to that for holes at the same filling.

Figure 4 shows the variation of the structure factor $S(\vec{Q})$ and the superfluid density $\rho_{s}$ as a function of the magnetization $m$, for two different values of $\tilde{g}$, i.e., 1.4 and 2.5. A key point to note here is that, in general, larger values of repulsion aid in the formation of a CDW, whereas larger values of NNN tunneling $t_{2}$ help a particle hop in the same sublattice. For $\tilde{g}=1.4$, at half-filling, the HCBs form a checkerboard solid shown in Fig. 5(a) and indicated by a peak in the structure factor $S(\pi, \pi)$. Slightly away from half-filling, a supersolid region develops after which the system retains only its superfluidity. The reason can be understood by examining the coeffi-
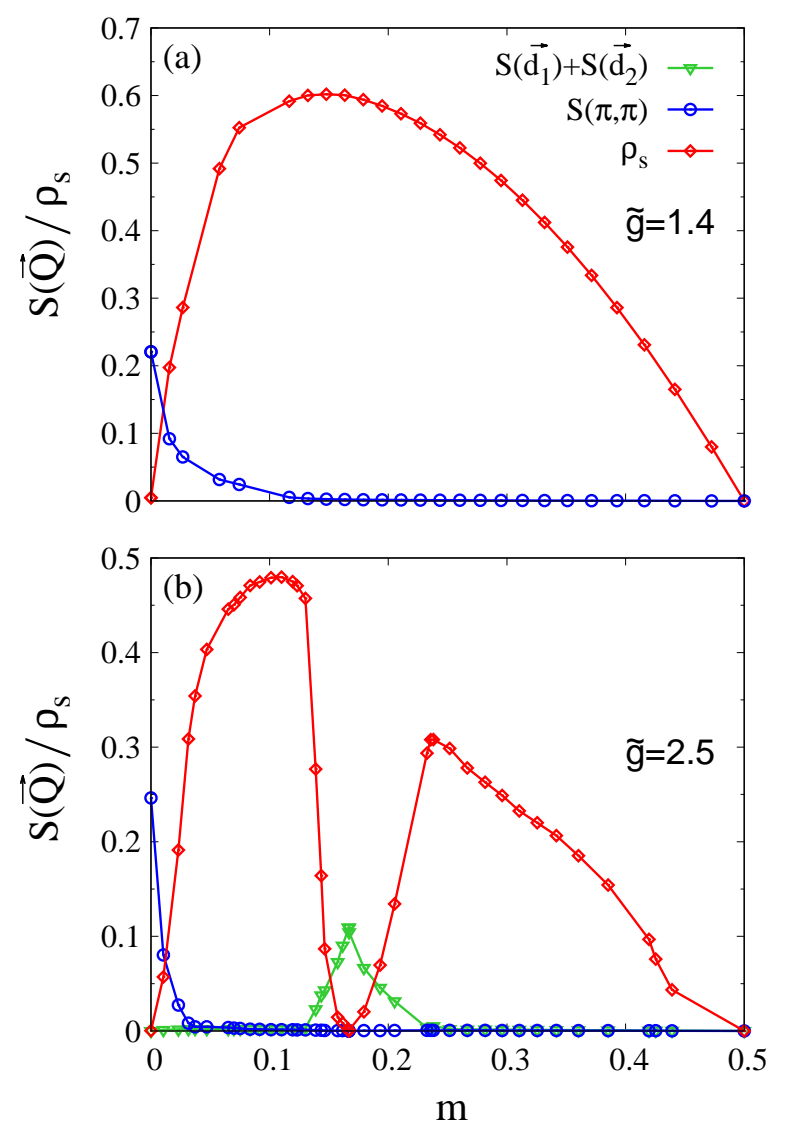

FIG. 4. (Color online) Plots of structure factor $S(\vec{Q})$ and superfluid density $\rho_{s}$ vs magnetization $m$ for HCBs on a $18 \times$ 18 lattice with $t / \omega_{0}=1.0$ and when (a) $\tilde{g}=1.4$ and (b) $\tilde{g}=2.5$. Curves are averaged results from simulations using three different random number seeds.

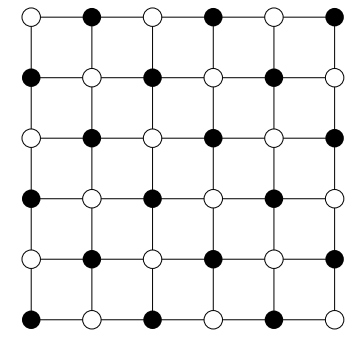

(a)

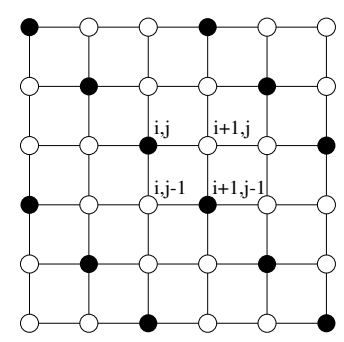

(b)

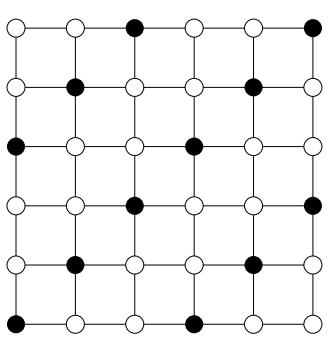

(c)
FIG. 5. Different types of CDWs: (a) checkerboard solid (cS) at half-filling with $S(\vec{Q})$ peaking at $\vec{Q}=(\pi, \pi)$; (b) diagonal striped solid (dsS) indicated by peak in $S(\vec{Q})$ at $\vec{Q}=(2 \pi / 3,2 \pi / 3)$; and (c) dsS characterized by ordering wavevector $\vec{Q}=(2 \pi / 3,4 \pi / 3)$.

cients of different terms in the Hamiltonian in Eq. (19). Since the NN repulsion dominates over the NNN and NNNN repulsions, at half-filling the system becomes a cS phase to avoid NN occupation, even though the particles experience NNN and NNNN repulsions. Now, if we add one additional particle to the half-filled system, the extra particle can be at any one of the empty sites; irrespective of the site it resides on, the particle will feel the same extra repulsion $4 V_{1}$. This extra particle can hop to its NNN or NNNN sites, without changing the repulsive interaction in the system which has a checkerboard solid in the background, resulting in the coexistence of superfluidity and CDW state. If we keep on increasing the particle number, after a certain filling-fraction, the checkerboard structure is lost with the system continuing to be a superfluid.

Now looking at Fig. 4(b) for $\tilde{g}=2.5$, we see that an additional CDW appears at fillings $\rho=1 / 3$ and $2 / 3$. Since the physics pertinent to $\rho=1 / 3$ is the same as that for $\rho=2 / 3$, we will analyze them interchangeably based on our convenience. At $\rho=1 / 3$, the HCBs form a diagonal striped solid manifesting spontaneously broken symmetry and characterized by a peak in the structure factor at wavevector $\overrightarrow{d_{1}}=(2 \pi / 3,2 \pi / 3)$ [corresponding to Fig. [5(b)] or $\overrightarrow{d_{2}}=(2 \pi / 3,4 \pi / 3)$ [related to Fig. [5(c)]. Although each particle in the stripe experiences a repulsion $2 V_{2}$, it is still the minimum energy state of the system at one-third filling. If we add one extra particle to the system, it occupies any one of the empty sites between the stripes and experiences a repulsion $2 V_{1}+V_{2}+2 V_{3}$. Now, this extra particle can hop to any of its unoccupied 
NN, NNN, or NNNN sites without a change in the potential energy of the system; thus, coexistence of stripe order and superfluidity is realized on the interstitial side. On the other hand, if we remove one particle from the system at $\rho=1 / 3$, the extra hole (residing in the stripes) can hop along the stripes without altering the potential energy; thus, supersolidity is exhibited on the other (i.e., vacancy) side of the diagonal striped phase as well. Thus, the mechanism governing the existence of a supersolid phase away from commensurate fillings $1 / 2$ and $1 / 3$, on our unfrustrated system (i.e., the square lattice), is that interstitials or vacancies can move without frustration, i.e., without a cost in the potential energy.

The complete ground state phase diagram is depicted in Fig. 6] The half-filled system shows the signature of a checkerboard solid (cS) for all $\tilde{g}$ values above 1.37 . Next to this CDW, we have a supersolid region (cSS) where $S(\pi, \pi)$ and $\rho_{s}$ coexist homogeneously. On the other hand, at filling fraction $\rho=1 / 3$, the system realizes a dsS beyond $\tilde{g}=2.11$. On both sides of this striped solid, we have a region of supersolid (dsSS) which is a homogeneous coexistence of the diagonal striped solid and a superfluid. As we increase $\tilde{g}$ beyond 1.37 , the width of the supersolid region cSS increases and attains its maximum at $\tilde{g}=2.0$. Further increase in $\tilde{g}$ results in a decrease in the width of the cSS region, thereby making way for diagonal stripe supersolid at higher values of $\tilde{g}$. However, we should point out that there is no direct supersolid-supersolid transition. Next, it is interesting to note that there is an asymmetry in the extent of the dsSS region around one-third filling. Thus, there is an asymmetry at $\rho=1 / 3$ with respect to doping with interstitials and vacancies similar to the asymmetry at one-fourth filling reported in Ref. 16 for a $t_{1}-V_{1}-V_{2}$ model when

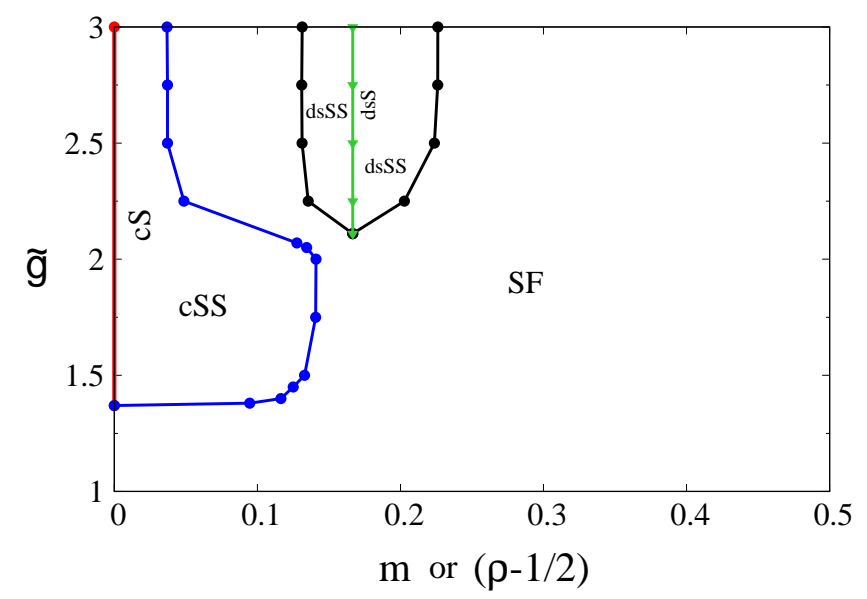

FIG. 6. (Color online) Phase diagram in terms of magnetization (or filling-fraction $\rho$ ) for $\mathrm{HCBs}$ on a $18 \times 18$ lattice with $t / \omega_{0}=1.0$. cS represents checkerboard solid with cSS being the corresponding supersolid; dsS stands for diagonal striped solid with dsSS being the related supersolid. Plots represent averaged results from simulations employing three different random number seeds.
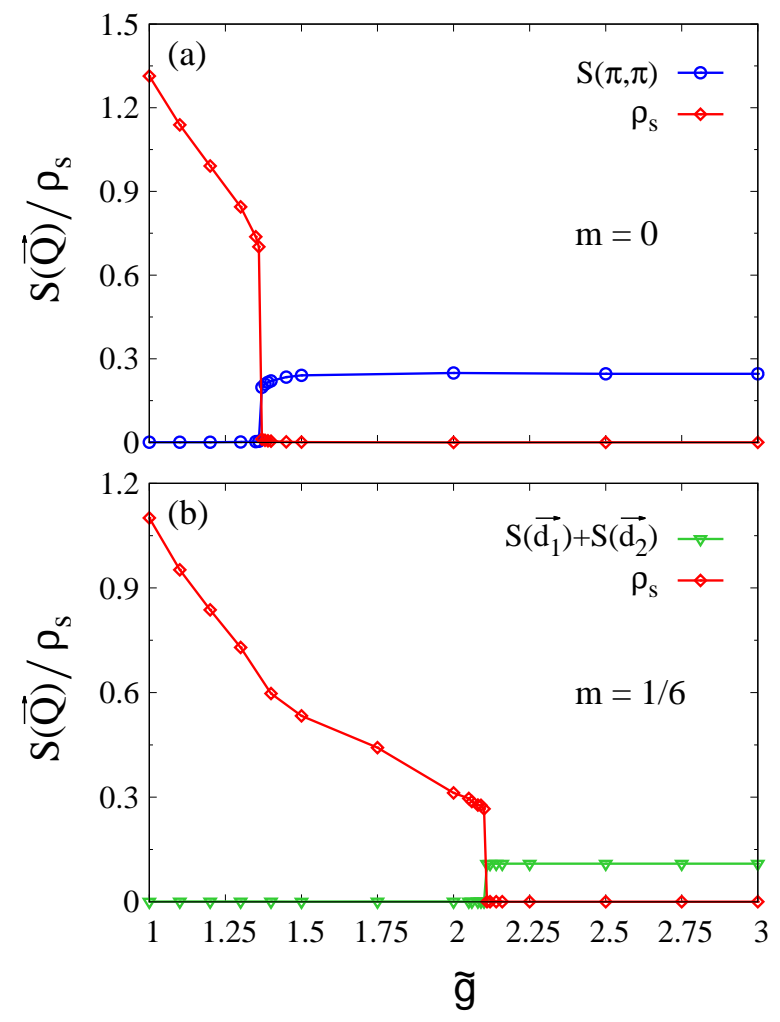

FIG. 7. (Color online) Plots of $S(\vec{Q})$ and $\rho_{s}$ vs coupling strength $\tilde{g}$ depicting first-order transitions at two different magnetization values: (a) $m=0$ (or half-filling) and (b) $m=$ $1 / 6$ (or two-third filling).

$V_{1}<2 V_{2}$. It is also worth noting that, at lower fillings such as $\rho=1 / 4$ and $\rho=1 / 5$, there is no CDW order.

In a recent study of HCBs on a square lattice with NN hopping and $\mathrm{NN}$ repulsion (i.e., in a $t_{1}-V_{1}$ model), when a sizeable external potential is applied along the diagonal stripes in Figs. 5(b) and 5(c), the authors obtain the corresponding diagonal striped $\mathrm{CDW}$ at $\rho=1 / 3$ and a striped supersolid phase away from one-third filling $\underline{54}$. Similar to our case, the physics governing the formation of a supersolid phase is that the interstitial particles or vacancies in the vicinity of the commensurate filling $\rho=$ $1 / 3$ can hop without changing the potential energy of the system.

In our simulations using SSE, we cannot tune the magnetization (density) directly. Instead, we tune the magnetic field which determines the magnetization of the system. For a particular value of the magnetic field, the resulting magnetization generally fluctuates during simulation. As a result, usually it is not possible to study the nature of the phase transitions by keeping the magnetization (filling-fraction) fixed at a particular value and varying $\tilde{g}$. However, when the system is in a CDW state, the magnetization remains constant over a range of magnetic field values; this makes it possible to vary $\tilde{g}$ at a fixed magnetization.

We see from Fig. 7(a) that for the half-filled system 
(i.e., at $m=0$ ), as we increase the $\tilde{g}$ value from 1 to 3 , the structure factor $S(\pi, \pi)$ jumps from 0 to almost its maximum value and the superfluid density suddenly drops down to zero at $\tilde{g}=1.37$. In the phase diagram (depicted in Fig 60), this indicates a first-order transition at $\tilde{g}=1.37$ from a superfluid to a checkerboard solid at filling-fraction $\frac{1}{2}$; since the transition is from a $\mathrm{U}(1)$ symmetry breaking state to a translational symmetry breaking state, the order of the transition is consistent with Landau's picture. Similarly at magnetization $m=\frac{1}{6}$ corresponding to filling fraction $\frac{2}{3}$, at $\tilde{g}=2.11$, Fig. [7(b) shows a dramatic jump in the structure factor $S(2 \pi / 3,2 \pi / 3)+S(2 \pi / 3,4 \pi / 3)$ from 0 to its maximum value accompanied by a discontinuous drop in the superfluid density to zero. This signifies a first-order transition as we move along the $\tilde{g}$-axis at $m=1 / 6$ in the phase diagram (shown in Fig 6). Thus, consistent with the literature $\underline{16}, 18,55$, no supersolidity is detected at commensurate fillings in our unfrustrated system.

In contrast to Fig. 7(a), Fig. (8) depicts the behavior of the order parameters (i.e., structure factor, superfluid density and magnetization) as we tune the magnetic field at the fixed value of coupling $\tilde{g}=2.5$. The continuous change in the order parameters as a function of magnetic field $h$ eliminates the possibility of a first-order phase transition. This further signifies that in the phase diagram (displayed in Fig [6), as we move along the $m$-axis at any particular $\tilde{g}$ value, all the different phases are separated from each other via continuous phase transitions, i.e., all supersolid-solid and superfluid-supersolid transitions are second order.

We will now identify the minimum model for the diagonal striped supersolid. Compared to the checkerboard supersolid, the dsSS phase is rarely observed. To determine the minimum model for the realization of the dsSS phase, we first identify the necessary repulsions required to observe the diagonal striped solid phase in the

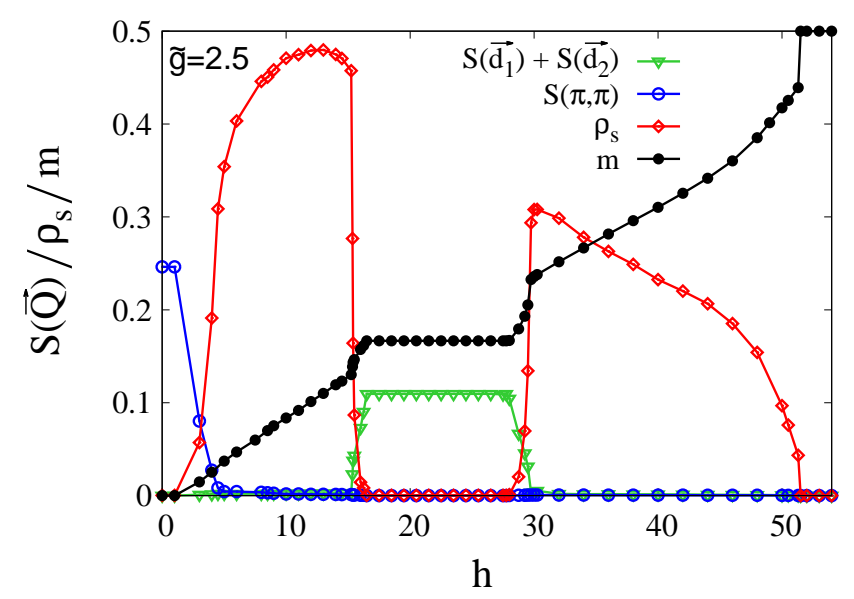

FIG. 8. (Color online) Evolution of order parameters $S(\vec{Q})$, $\rho_{s}$ and $m$ as the magnetic field $h$ is varied at a fixed coupling strength $\tilde{g}=2.5$. No discontinuous transitions are exhibited.

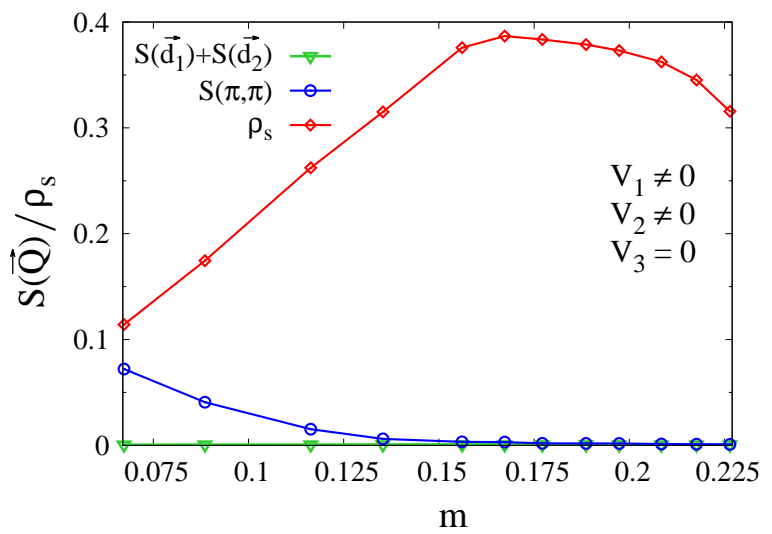

FIG. 9. (Color online) Variation of $S(\vec{Q})$ and $\rho_{s}$ vs magnetization $m$ in the absence of the NNNN repulsion $V_{3}$ along $\mathrm{x}$ - and $\mathrm{y}$-axes in the $t_{1}-t_{2}-t_{3}-V_{1}-V_{2}-V_{3}$ model of Eq. (18).

$t_{1}-t_{2}-t_{3}-V_{1}-V_{2}-V_{3}$ model of Eq. (18). From Fig. (9), we see that, as soon as we tune the NNNN repulsion $V_{3}$ along x and y-axes to zero, the structure factor corresponding to the dsS phase completely disappears. This feature can be explained based on Figs. 5(b) and 5 (c). For instance, when the NNNN repulsion $V_{3}$ is set to zero in the structure given by Fig. 5(b), the particles at sites $(\mathrm{i}, \mathrm{j})$ and $(\mathrm{i}+1, \mathrm{j}-1)$ can both be shifted to the neighboring sites $(i+1, j)$ and $(i, j-1)$ without changing the potential energy of the system. This process destroys the striped structure. Thus, it follows that all the three repulsions (i.e., $V_{1}, V_{2}$, and $V_{3}$ ) are necessary to stabilize the dsS structure. A similar argument can be made to destroy the structure given by Fig. 5 (c).

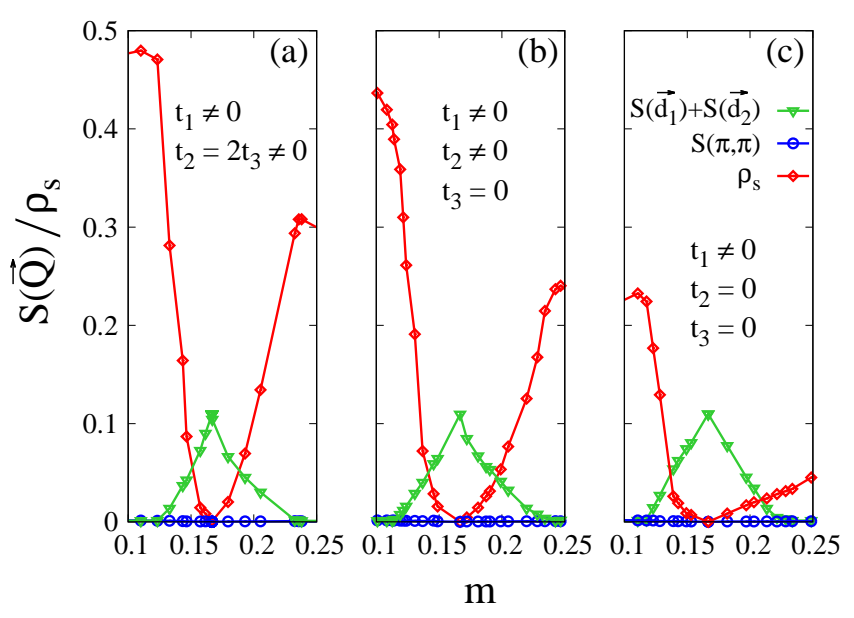

FIG. 10. (Color online) Plots of $S(\vec{Q})$ and $\rho_{s}$ vs magnetization $m$, in the vicinity of striped phase, for three different cases in the $t_{1}-t_{2}-t_{3}-V_{1}-V_{2}-V_{3}$ model of Eq. (18): (a) all the three hoppings $t_{1}, t_{2}$, and $t_{3}$ are present; (b) NNNN hopping $t_{3}$ along $\mathrm{x}$ - and $\mathrm{y}$-axes is set to zero; and (c) only $\mathrm{NN}$ hopping $t_{1}$ is present. The minimum model for diagonal striped solid (dsS) is shown to be $t_{1}-V_{1}-V_{2}-V_{3}$. 
Next, in Fig. 10 we focus on the region in the vicinity of the striped phase. Compared to Fig. 10(a), in which all the three hopping parameters are non-zero, the superfluid density reduces slightly when the NNNN hopping $t_{3}$ is set to zero [as can be seen in Fig. 10(b)]. The interesting feature to note is that, even when only NN hopping $t_{1}$ is present with the other two hopping parameters $t_{2}$ and $t_{3}$ being zero [as in Fig. 10(c)], we have a diagonal striped supersolid region around $m=1 / 6$ with the width of the dsSS being almost unaffected. This elucidates the fact that the minimum model to obtain a dsSS phase is the $t_{1}-V_{1}-V_{2}-V_{3}$ model.

\section{COMPARISON WITH LSNO EXPERIMENTAL RESULTS}

Stripe-like charge order has been reported in a number of layered transition-metal oxides ${ }^{35}$. Among these compounds, the layered nickelate LSNO is an archetypal system to exhibit a firm charge stripe order. In $\mathrm{La}_{2-x} \mathrm{Sr}_{\mathrm{x}} \mathrm{NiO}_{4}$, static checkerboard charge order [such as in Fig. [5(a)] is expressed at $x=1 / 2$ and static diagonal stripe order [as shown in Figs. 5(b) and 5(c)] is manifested at $x=1 / 3$ with the transition temperatures at these dopings showing local maxima ${ }^{36-41}$. The observed lattice constant ratio c/a in LSNO displays a maximum at $x=1 / 2$, thereby indicating that in the region $0<x<1 / 2$ holes are doped into the $d_{x^{2}-y^{2}}$ orbitals and in the region $1 / 2<x<1$ holes are doped into the $d_{z^{2}}$ orbitals 38,56 . Measurements of Hall coefficient for $\mathrm{La}_{2-\mathrm{x}} \mathrm{Sr}_{\mathrm{x}} \mathrm{NiO}_{4}$ by T. Katsufuji et al $\frac{57}{5}$, revealed that the charge carriers change from electron-like to hole-like while going from the hole density $x<1 / 3$ to $x>1 / 3$.

In the undoped $\mathrm{La}_{2} \mathrm{NiO}_{4}$, the oxidation state of nickel is $\mathrm{Ni}^{2+}$ with the electronic configuration $[\mathrm{Ar}] 4 \mathrm{~s}^{0} 3 \mathrm{~d}^{8}$. Hence, only $d_{z^{2}}$ and $d_{x^{2}-y^{2}}$ orbitals are relevant in the doped compound $\mathrm{La}_{2-\mathrm{x}} \mathrm{Sr}_{\mathrm{x}} \mathrm{NiO}_{4}$. The electron-phonon interaction term of the Hamiltonian is given by

$$
H_{e p}=-\frac{g \omega_{0}}{4} \sum_{i, j}\left(d_{z^{2} ; i, j}^{\dagger} d_{x^{2}-y^{2} ; i, j}^{\dagger}\right)\left(\begin{array}{cc}
q_{x ; i, j}+q_{y ; i, j}+4 q_{z ; i, j} & -\sqrt{3} q_{x ; i, j}+\sqrt{3} q_{y ; i, j} \\
-\sqrt{3} q_{x ; i, j}+\sqrt{3} q_{y ; i, j} & 3 q_{x ; i, j}+3 q_{y ; i, j}
\end{array}\right)\left(\begin{array}{c}
d_{z^{2} ; i, j} \\
d_{x^{2}-y^{2} ; i, j}
\end{array}\right)
$$

where the distortions $q_{x ; i, j} \equiv\left(a_{x ; i, j}^{\dagger}+a_{x ; i, j}\right)-\left(a_{x ; i-1, j}^{\dagger}+\right.$ $\left.a_{x ; i-1, j}\right), q_{y ; i, j} \equiv\left(b_{y ; i, j}^{\dagger}+b_{y ; i, j}\right)-\left(b_{y ; i, j-1}^{\dagger}+b_{y ; i, j-1}\right)$, and $q_{z ; i, j} \equiv\left(c_{z ; i, j}^{\dagger}+c_{z ; i, j}\right)$. In the undoped compound, since both $d_{z^{2}}$ and $d_{x^{2}-y^{2}}$ orbitals are occupied, there are only breathing mode distortions $\left(4 q_{x ; i, j}+4 q_{y ; i, j}+4 q_{z ; i, j}\right)$ and no active Jahn-Teller (JT) distortions. Now, when we introduce holes in the system (by doping with $\mathrm{Sr}$ such that $0<x<1 / 2$ ), the holes occupy the $d_{x^{2}-y^{2}}$ orbitals; this is because a site with a single electron in $d_{x^{2}-y^{2}}$ orbital will produce in-plane distortions $\left(3 q_{x}+3 q_{y}\right)$ which have a greater incompatibility with the breathing mode distortions $\left(4 q_{x}+4 q_{y}+4 q_{z}\right)$ on the adjacent sites and thus cost more energy than a singly occupied $d_{z^{2}}$ orbital. These $d_{x^{2}-y^{2}}$ holes can hop and are responsible for the transport properties. Each site with a $d_{x^{2}-y^{2}}$ hole is JT active.

The Hamiltonian for cooperative Jahn-Teller (CJT) distortions in the two-dimensional LSNO system involves holes in $d_{x^{2}-y^{2}}$ orbitals as the active carriers. The starting Hamiltonian $H_{\text {LSNO }}$, describing $\mathrm{La}_{2-\mathrm{x}} \mathrm{Sr}_{\mathrm{x}} \mathrm{NiO}_{4}$ for $0 \leq \mathrm{x} \leq 0.5$, consists of the following terms expressed in terms of the creation (destruction) operator $h_{i, j}^{\dagger}\left(h_{i, j}\right)$ for the holes in $d_{x^{2}-y^{2}}$ orbitals.

(i) Hopping term

$$
H_{t}^{\prime}=\frac{3 t}{4} \sum_{i, j}\left(h_{i+1, j}^{\dagger} h_{i, j}+h_{i, j+1}^{\dagger} h_{i, j}+\text { H.c. }\right)
$$

(ii) hole-phonon interaction term:

$$
\begin{aligned}
H_{I}^{\prime}=\frac{3}{4} g \omega_{0} & \sum_{i, j}\left[\left(a_{x ; i, j}^{\dagger}+a_{x ; i, j}\right)\left(n_{i, j}^{h}-n_{i+1, j}^{h}\right)\right. \\
& \left.+\left(b_{y ; i, j}^{\dagger}+b_{y ; i, j}\right)\left(n_{i, j}^{h}-n_{i, j+1}^{h}\right)\right]
\end{aligned}
$$

and (iii) lattice term:

$$
H_{l}^{\prime}=\omega_{0} \sum_{i, j}\left(a_{x ; i, j}^{\dagger} a_{x ; i, j}+b_{y ; i, j}^{\dagger} b_{y ; i, j}\right)
$$

where $n_{i, j}^{h} \equiv h_{i, j}^{\dagger} h_{i, j}$.

The Lang-Firsov transformed Hamiltonian is given by $\tilde{H}_{\mathrm{LSNO}}=\exp (S) H_{\mathrm{LSNO}} \exp (-S)$ where $S$ has the form

$$
\begin{aligned}
S=\frac{3}{4} g \sum_{i, j} & {\left[\left(a_{x ; i, j}^{\dagger}-a_{x ; i, j}\right)\left(n_{i, j}^{h}-n_{i+1, j}^{h}\right)\right.} \\
& \left.+\left(b_{y ; i, j}^{\dagger}-b_{y ; i, j}\right)\left(n_{i, j}^{h}-n_{i, j+1}^{h}\right)\right] .
\end{aligned}
$$

Setting $t^{\prime}=-3 t / 4$ and $g^{\prime}=-3 g / 4$, in the nonadiabatic regime $\left(\left|t^{\prime}\right| / \omega_{0} \leqslant 1\right)$ and at strong coupling (i.e., large $g^{\prime 2}$ ), the transformed Hamiltonian can be split into two terms: the unperturbed Hamiltonian and the perturbation term. These two terms are the same as the ones given by Eqs. (5) and (6), except that they are written in hole-operator language; both $\gamma$ and $\eta$ are set to zero value; and $t$ and $g$ are replaced by $t^{\prime}$ and $g^{\prime}$, respectively. If the carriers are taken to be HCBs instead 
of fermionic holes, then after following the same secondorder perturbative procedure as in Sec. III, we end up with an effective Hamiltonian that is exactly the same as that given by Eq. (18) with $\gamma=0=\eta$ and with $t$ and $g$ being replaced by $t^{\prime}$ and $g^{\prime}$, respectively. It is important to note that the small parameter value is again given by $\left[\frac{t^{2}}{2\left(E_{p}+V_{p}\right) \omega_{0}}\right]^{\frac{1}{2}}$ and remains unaltered. Now, since we are dealing with fermionic holes and not HCBs, we get the following effective Hamiltonian:

$$
\begin{aligned}
H_{\mathrm{eff}}^{\prime}= & -\left(E_{p}+2 V_{z}\right) \sum_{i, j} n_{i, j}^{h} \\
& -t_{1} \sum_{i, j}\left(h_{i+1, j}^{\dagger} h_{i, j}+h_{i, j+1}^{\dagger} h_{i, j}+\text { H.c. }\right) \\
& +V_{1} \sum_{i, j}\left(n_{i, j}^{h} n_{i+1, j}^{h}+n_{i, j}^{h} n_{i, j+1}^{h}\right) \\
& -t_{2} \sum_{i, j}\left(h_{i+1, j+1}^{\dagger}\left(1-n_{i+1, j}^{h}-n_{i, j+1}^{h}\right) h_{i, j}\right. \\
& \left.+h_{i-1, j+1}^{\dagger}\left(1-n_{i-1, j}^{h}-n_{i, j+1}^{h}\right) h_{i, j}+\text { H.c. }\right) \\
& +V_{2} \sum_{i, j}\left(n_{i, j}^{h} n_{i+1, j+1}^{h}+n_{i, j}^{h} n_{i-1, j+1}^{h}\right) \\
& -t_{3} \sum_{i, j}\left(h_{i+2, j}^{\dagger}\left(1-2 n_{i+1, j}^{h}\right) h_{i, j}\right. \\
& \left.+h_{i, j+2}^{\dagger}\left(1-2 n_{i, j+1}^{h}\right) h_{i, j}+\text { H.c. }\right) \\
& +V_{3} \sum_{i, j}\left(n_{i, j}^{h} n_{i+2, j}^{h}+n_{i, j}^{h} n_{i, j+2}^{h}\right),
\end{aligned}
$$

with $\gamma=0=\eta$ and with $t$ and $g$ being replaced by $t^{\prime}$ and $g^{\prime}$, respectively. Since, the interaction terms for the CJT Hamiltonian of LSNO are the same as those for the $t_{1}-t_{2}-t_{3}-V_{1}-V_{2}-V_{3}$ Hamiltonian in Eq. (18), in LSNO also we expect to get the same charge-ordered phases obtained for the $t_{1}-t_{2}-t_{3}-V_{1}-V_{2}-V_{3}$ model. Thus. at hole doping $1 / 2$ and $1 / 3$ (i.e., at $x=1 / 2$ and $x=1 / 3$ in $\mathrm{La}_{2-\mathrm{x}} \mathrm{Sr}_{\mathrm{x}} \mathrm{NiO}_{4}$ ), we will realize checkerboard solid and diagonal stripes, respectively, which match exactly with the charge ordering obtained for LSNO experimentally.

Now, if we add one extra hole to the system at onethird hole doping (i.e., at $x=1 / 3$ ), then the extra hole will reside in the region between two diagonal stripes. This extra hole can hop anywhere in the region between the stripes without changing the potential energy of the system. Thus, the carriers for the hole doping $x>1 / 3$ are holes. On the other hand, adding one electron to the striped phase so that $x<1 / 3$ will result in the extra electron occupying any one of the sites along the stripes; this extra electron is free to hop along the stripes without altering the potential energy of the system. This means that electrons are the carriers for the doping $x<1 / 3$. Therefore, based on our model we can explain the hole or electron doping (into the charge-ordered Mott insulator $\mathrm{La}_{5 / 3} \mathrm{Sr}_{1 / 3} \mathrm{NiO}_{4}$ ) that was reported by $\mathrm{T}$. Katsufuji et $a l .57$
One can obviously ask how a system of HCBs can reproduce some experimental results of a system of electrons. The reason behind the charge orderings at holedoping values $1 / 2$ and $1 / 3$ is repulsion; hopping does not play any role in the ordering. Hence, for these two CDWs, it does not matter whether the carriers of the system are HCBs or electrons. Close to one-third doping, only single carrier physics plays a role; consequently, particle-hole asymmetry is captured. Next, it is important to note that CJT interaction is needed to generate NNN and NNNN repulsions $V_{2}$ and $V_{3}$ which in turn are needed to explain diagonal stripes. Thus, we see that our work resolves the controversy whether cooperative Jahn-Teller distortions can explain the observed diagonal-stripe charge order at one-third doping in LSNO43-45. Lastly, it should also be pointed out that, although experimentally ${ }^{38}$ insulating behavior is observed in LSNO for $x \lesssim 0.9$, theoretically we expect metallic nature; we believe, this discrepancy is due to localization effects produced by disorder in real LSNO systems.

\section{CONCLUSIONS AND OPEN PROBLEMS}

To conclude, we investigated a 2D system of HCBs, modulated by the cooperative breathing mode, which is important in real materials such as $\mathrm{BaBiO}_{3}$ and nickelates as well as in artificial cold-atom systems. Using a duality treatment, we obtained the effective Hamiltonian and generated the phase diagram employing the SSE technique.

In the phase diagram displayed in Fig. 6, a first-order transition occurs from a superfluid to a checkerboard solid at filling-fraction $1 / 2$ and from a superfluid to a diagonal striped solid at filling $1 / 3$. We interpreted the nature of the transition by invoking Landau's explanation. It would be interesting to verify whether in other unfrustrated lattices, such as the checkerboard lattice, a discontinuous superfluid-solid transition is manifested at commensurate fillings such as $1 / 4^{55}$. Furthermore, at a fixed interaction strength, our $t_{1}-t_{2}-t_{3}-V_{1}-$ $V_{2}-V_{3}$ model realizes only continuous transitions (i.e., superfluid-supersolid and supersolid-solid transitions) as density is varied. Contrastingly, the $t_{1}-t_{2}-t_{3}-V_{1}$ model (pertaining to the strong-coupling case of the Holstein model) manifests a discontinuous superfluid-supersolid transition when density is varied ${ }^{21}$. Thus, more insight is needed to identify which class of models yield what type of superfluid-supersolid transition.

We have identified the $t_{1}-V_{1}-V_{2}-V_{3}$ model as the minimum model for obtaining a diagonal striped supersolid on a square lattice. It would be exciting to realize this system in a cold-atom system, thereby adding to the understanding of lattice supersolidity generated by longrange interactions 13 .

The asymmetry of the supersolid phase about a commensurate filling, such as one third in our case and one fourth in the case of Ref. 16, in a square lattice oc- 
curs possibly because particle-hole symmetry is not respected by the Hamiltonian about these fillings. It would be worthwhile to study the nature of such asymmetry in other lattices such as honeycomb, checkerboard, etc.

We have explained the charge ordering in $\mathrm{La}_{2-x} \mathrm{Sr}_{x} \mathrm{NiO}_{4}$ at hole-doping $x=1 / 2$ and $1 / 3$ by considering cooperative Jahn-Teller effect. However, studies involving CJT effect are needed at dopings away from these fillings and particularly in the region $x>1 / 2$ where holes are doped into the $d_{z^{2}}$ orbitals. Also of interest would the explanation for the metal-insulator transition observed at $x \sim 0.938$.

In a different but related system $\mathrm{La}_{2-\mathrm{x}} \mathrm{Sr}_{\mathrm{x}} \mathrm{CoO}_{4}$, CDWs similar to those in LSNO are observed. At half doping, there is a signature of checkerboard charge ordering with alternate $\mathrm{Co}^{2+}$ and $\mathrm{Co}^{3+}$ ions (below $T_{\mathrm{CO}} \approx$ $750 \mathrm{~K})^{58}$. On the other hand, at the doping $x=1 / 3$, the holes form a diagonal-stripe pattern similar to the stripes in LSNO at a transition temperature well above the room temperature ${ }^{59}-62$. Furthermore, the presence of substantial disorder in these diagonal stripes has been confirmed by the experiment ${ }^{62}$ done by A. T. Boothroyd et al. The electronic configuration of cobalt is $[\mathrm{Ar}] 3 \mathrm{~d}^{7} 4 \mathrm{~s}^{2}$. In $\mathrm{La}_{2-x} \mathrm{Sr}_{\mathrm{x}} \mathrm{CoO}_{4}$, cobalt shows two different oxidation states: $\mathrm{Co}^{2+}$ and $\mathrm{Co}^{3+}$. The $\mathrm{Co}^{3+}$ ions are found to have the low-spin ground state $(\mathrm{S}=0)^{63}$ with the electronic configuration $[\mathrm{Ar}] 3 \mathrm{~d}^{6}$. In this case, all the six $d$ electrons occupy the $t_{2 g}$ orbitals and both the $e_{g}$ orbitals are empty. Therefore, $\mathrm{Co}^{3+}$ ions do not cause any JahnTeller distortion in the system. On the other hand, in the case of $\mathrm{Co}^{2+}$ ions, the electrons are in the high-spin ground state $(\mathrm{S}=3 / 2)$ with the electronic configuration $[\mathrm{Ar}] 3 \mathrm{~d}^{7}$. This state consists of five electrons in the $t_{2 g}$ orbitals and two in the $e_{g}$ orbitals. Two out of the three $t_{2 g}$ orbitals are completely filled with four electrons, whereas the remaining orbital contains a single electron. Since both the $e_{g}$ orbitals are occupied by one electron each, JT distortion comes into play due to the singly occupied $t_{2 g}$ orbital only. Owing to the fact that the JT distortion arising from $t_{2 g}$ electrons is weaker than the one arising from $e_{g}$ electrons, it needs to be examined whether this can explain the disorder in the stripe pattern in $\mathrm{La}_{2-\mathrm{x}} \mathrm{Sr}_{\mathrm{x}} \mathrm{CoO}_{4}$.

\section{ACKNOWLEDGEMENTS}

The computing resources of the Condensed Matter Physics Division (Saha Institute of Nuclear Physics) have been used extensively. Valuable discussions with R. Pankaj are acknowledged. A.G. would especially like to thank S. Kar, G. Majumdar and M. Sarkar for useful discussions regarding SSE. S.Y. thanks P. B. Littlewood for stimulating discussions and Cavendish lab for hospitality during the initial stages of this work.

\section{Appendix A: Nearest-neighbor repulsion}

The second order perturbation term is given by

$$
\begin{aligned}
& H^{(2)}=-\sum_{m} \sum_{i, j, k, l} \frac{\left\langle\left. 0\right|_{p h} H_{1 i, j} \mid m\right\rangle_{p h}\left\langle\left. m\right|_{p h} H_{1 k, l} \mid 0\right\rangle_{p h}}{E_{0}^{p h}-E_{m}^{p h}} \\
&=-t_{1}^{2} \sum_{m} \sum_{i, j, k, l} \frac{1}{\Delta E_{m}^{p h}}\left[\left(d_{i+1, j}^{\dagger} d_{i, j}\left\langle\left. 0\right|_{p h}\left(\tau_{-x}^{i j}-1\right) \mid m\right\rangle_{p h}\right.\right. \\
&+d_{i, j}^{\dagger} d_{i+1, j}\left\langle\left. 0\right|_{p h}\left(\tau_{+x}^{i j}-1\right) \mid m\right\rangle_{p h} \\
&+d_{i, j+1}^{\dagger} d_{i, j}\left\langle\left. 0\right|_{p h}\left(\tau_{-y}^{i j}-1\right) \mid m\right\rangle_{p h} \\
&\left.+d_{i, j}^{\dagger} d_{i, j+1}\left\langle\left. 0\right|_{p h}\left(\tau_{+y}^{i j}-1\right) \mid m\right\rangle_{p h}\right) \\
& \times\left(d_{k+1, l}^{\dagger} d_{k, l}\left\langle\left. m\right|_{p h}\left(\tau_{+x}^{k l}{ }^{\dagger}-1\right) \mid 0\right\rangle_{p h}\right. \\
&+d_{k, l}^{\dagger} d_{k+1, l}\left\langle\left. m\right|_{p h}\left(\tau_{-x}^{k l}{ }^{\dagger}-1\right)\right)|0\rangle_{p h} \\
&+d_{k, l+1}^{\dagger} d_{k, l}\left\langle\left. m\right|_{p h}\left(\tau_{+y}^{k l}{ }^{\dagger}-1\right)\right)|0\rangle_{p h} \\
&\left.\left.+d_{k, l}^{\dagger} d_{k, l+1}\left\langle\left. m\right|_{p h}\left(\tau_{-y}^{k l}{ }^{\dagger}-1\right)\right)|0\rangle_{p h}\right)\right],
\end{aligned}
$$

where $t_{1}=t e^{-\left(E_{p}+V_{p}\right) / \omega_{0}}$ and $\Delta E_{m}^{p h}=E_{0}^{p h}-E_{m}^{p h}$.

As already mentioned in Sec. IA the NN repulsion results from a process where a particle hops to its neighboring site and returns back, which in $2 \mathrm{D}$ consists of two terms: $\sum_{i, j}\left[n_{i, j}\left(1-n_{i+1, j}\right)+n_{i+1, j}\left(1-n_{i, j}\right)\right]$ and $\sum_{i, j}\left[n_{i, j}\left(1-n_{i, j+1}\right)+n_{i, j+1}\left(1-n_{i, j}\right)\right]$.

Since, $\sum_{i, j} n_{i, j}\left(1-n_{i+1, j}\right)=\sum_{i, j} n_{i+1, j}\left(1-n_{i, j}\right)$ and $\sum_{i, j} n_{i, j}\left(1-n_{i, j+1}\right)=\sum_{i, j} n_{i, j+1}(1-$ $\left.n_{i, j}\right)$, so the process is effectively given by $\sum_{i, j}\left[n_{i, j}\left(1-n_{i+1, j}\right)+n_{i, j}\left(1-n_{i, j+1}\right)\right]$ with the coefficient being twice.

Now, we can rewrite the term $\sum_{i, j} n_{i, j}\left(1-n_{i+1, j}\right)$ as

$$
\begin{aligned}
\sum_{i, j} d_{i, j}^{\dagger} d_{i, j}\left(1-d_{i+1, j}^{\dagger} d_{i+1, j}\right) & =\sum_{i, j} d_{i, j}^{\dagger} d_{i, j} d_{i+1, j} d_{i+1, j}^{\dagger} \\
& =\sum_{i, j} d_{i, j}^{\dagger} d_{i+1, j} d_{i+1, j}^{\dagger} d_{i, j}
\end{aligned}
$$

Looking at the expression of $H^{(2)}$, one can figure out that the above term comes from the multiplication of the terms $d_{i, j}^{\dagger} d_{i+1, j}$ and $d_{k+1, l}^{\dagger} d_{k, l}$ for $k=i$ and $l=j$. So, the coefficient of this term is given by

$$
t_{1}^{2} \sum_{m} \frac{\left\langle\left. 0\right|_{p h}\left(\tau_{+x}^{i j}-1\right) \mid m\right\rangle_{p h}\left\langle\left. m\right|_{p h}\left(\tau_{+x}^{i j \dagger}-1\right) \mid 0\right\rangle_{p h}}{\Delta E_{m}^{p h}}
$$

where

$$
\begin{aligned}
& \tau_{+x}^{i j}=\exp \left[g\left(2 a_{i, j}-a_{i-1, j}-a_{i+1, j}\right)\right. \\
& \left.+g\left(b_{i+1, j-1}+b_{i, j}-b_{i, j-1}-b_{i+1, j}\right)+\gamma g\left(c_{i, j}-c_{i+1, j}\right)\right]
\end{aligned}
$$


consequently, the coefficient simplifies exactly to be $\frac{t_{1}^{2}}{\omega_{0}} G_{9}\left(4,1,1,1,1,1,1, \gamma^{2}, \gamma^{2}\right)$. Now, the general form $G_{n}\left(\alpha_{1}, \alpha_{2}, \cdots, \alpha_{n}\right)$ can be expressed as

$G_{n}\left(\alpha_{1}, \alpha_{2}, \cdots, \alpha_{n}\right)=\sum_{m_{1}, m_{2}, \ldots, m_{n}}^{\prime} \frac{\left(\alpha_{1} g^{2}\right)^{m_{1}} \cdots\left(\alpha_{n} g^{2}\right)^{m_{n}}}{m_{1} ! \cdots m_{n} !\left(m_{1}+\cdots+m_{n}\right)}$,

where $m_{i}=0,1,2, \ldots, \infty$ and the prime in $\sum^{\prime}$ implies the case $m_{1}=m_{2}=\ldots=m_{n}=0$ is excluded from the summation. It is important to note that for large values of $g^{2}, G_{n}$ can be approximately expressed as

$$
G_{n}\left(\alpha_{1}, \alpha_{2}, \cdots, \alpha_{n}\right) \approx \frac{\exp \left(\sum_{i=1}^{n} \alpha_{i} g^{2}\right)}{\sum_{i=1}^{n} \alpha_{i} g^{2}} .
$$

Then, the NN repulsion is given by

$$
-V_{z} \sum_{i, j}\left[n_{i, j}\left(1-n_{i+1, j}\right)+n_{i, j}\left(1-n_{i, j+1}\right)\right]
$$

where

$$
\begin{aligned}
V_{z} & =\frac{2 t^{2} e^{-2\left(E_{p}+V_{p}\right) / \omega_{0}}}{\omega_{0}} G_{9}\left(4,1,1,1,1,1,1, \gamma^{2}, \gamma^{2}\right) \\
& \approx \frac{2 t^{2}}{2 E_{p}+2 V_{p}} .
\end{aligned}
$$

Now, in arriving at Eq. (A4), we did not take into account the occupancy of the neighbors of the intermediate site. For example, when the particle hops from site $(i, j)$ to NN site $(i+1, j)$ and back, we have not considered the occupancy of the sites $(i+2, j),(i+1, j+1)$ and $(i+1, j-1)$, which are the neighboring sites of the intermediate site $(i+1, j)$ (as can be seen from Fig. 1). We will consider this occupancy in the next Appendix.

\section{Appendix B: NNN repulsion and NNNN repulsion}

In this appendix we first outline the procedure of calculating the coefficient of next-nearest-neighbor (NNN) repulsion which occurs along the diagonals. Consider the case where a particle hops to its neighboring site and returns back yielding the term $\propto \sum_{<i, j>} n_{i}\left(1-n_{j}\right)$ with $<i, j>$ indicating nearest-neighbor (NN) pairs of sites. In this process we have to take into account the occupancy of the neighboring sites of the intermediate site $j$. For example, in Fig. 11, if a particle at site 1 hops to site 2 and comes back, then the coefficient of this process depends on the occupancy of the sites 3,4 , and 5 . If all the three sites are empty, then this term can be expressed as $-V_{z} n_{1}\left(1-n_{3}\right)\left(1-n_{4}\right)\left(1-n_{5}\right)$ where $V_{z} \approx \frac{2 t^{2}}{2 E_{p}+2 V_{p}}$; here, we have omitted the term $\left(1-n_{2}\right)$ because the possibility of NN occupancy (for particle at site 1) is already

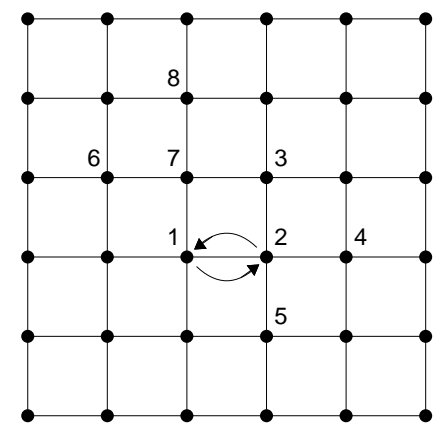

FIG. 11. Pictorial description of the process where a particle at site 1 hops to site 2 and comes back.

excluded from the process due to the large value of NN repulsion $2 V_{p}$. Due to numerical difficulties in our simulations using SSE, we need to simplify the four-operator term into a two-operator one by applying mean field to the remaining two operators. One can easily see that this mean-field procedure leaves us with a term which represents NNN repulsion (which acts along the diagonals) or NNNN repulsion (which acts along the axes).

We will now calculate the NNN repulsion coefficient which pertains to the diagonals of the square lattice in Fig. 11. To this end, we consider all the possible processes yielding the operator $n_{1} n_{3}$ and add all the corresponding terms to evaluate its coefficient. The following are the relevant cases.

Case 1: NNN interaction, when all the three neighboring sites of the intermediate site are unoccupied, involves the following.

(i) The contribution of particle hopping from site 1 to site 2 and coming back:

$$
\begin{aligned}
& =-\frac{2 t^{2}}{\left(2 E_{p}+2 V_{p}\right)} n_{1}\left(1-n_{3}\right)\left(1-n_{4}\right)\left(1-n_{5}\right) \\
& \approx-\frac{2 t^{2}}{\left(2 E_{p}+2 V_{p}\right)} n_{1}\left(1-n_{3}\right)\left\langle 1-n_{4}\right\rangle\left\langle 1-n_{5}\right\rangle \\
& \approx-\frac{2 t^{2}}{\left(2 E_{p}+2 V_{p}\right)} n_{1}\left(1-n_{3}\right)\left(\frac{1}{2}-m\right)^{2},
\end{aligned}
$$

where $\langle\ldots\rangle$ implies mean value and $\left\langle 1-n_{4}\right\rangle=\left\langle 1-n_{5}\right\rangle=$ $\left(\frac{1}{2}-m\right)$ with $m$ being the magnetization of the system.

(ii) The contribution of particle hopping from site 1 to site 7 and returning back involves a similar situation as (i) and is given as:

$$
\begin{aligned}
& \approx-\frac{2 t^{2}}{\left(2 E_{p}+2 V_{p}\right)} n_{1}\left(1-n_{3}\right)\left\langle 1-n_{6}\right\rangle\left\langle 1-n_{8}\right\rangle \\
& \approx-\frac{2 t^{2}}{\left(2 E_{p}+2 V_{p}\right)} n_{1}\left(1-n_{3}\right)\left(\frac{1}{2}-m\right)^{2} .
\end{aligned}
$$

(iii) The contribution of particle hopping from site 3 to 
site 2 and coming back:

$$
\begin{aligned}
& \approx-\frac{2 t^{2}}{\left(2 E_{p}+2 V_{p}\right)} n_{3}\left(1-n_{1}\right)\left\langle 1-n_{4}\right\rangle\left\langle 1-n_{5}\right\rangle \\
& \approx-\frac{2 t^{2}}{\left(2 E_{p}+2 V_{p}\right)} n_{3}\left(1-n_{1}\right)\left(\frac{1}{2}-m\right)^{2} .
\end{aligned}
$$

(iv) The particle hopping from site 3 to site 7 and returning back is similar to (iii) and yields the same expression as Eq. (B3).

(v) The contribution of particle hopping from site 4 to site 2 and coming back:

$$
\begin{aligned}
& \approx-\frac{2 t^{2}}{\left(2 E_{p}+2 V_{p}\right)}\left(1-n_{3}\right)\left(1-n_{1}\right)\left\langle n_{4}\right\rangle\left\langle 1-n_{5}\right\rangle \\
& \approx-\frac{2 t^{2}}{\left(2 E_{p}+2 V_{p}\right)}\left(1-n_{3}\right)\left(1-n_{1}\right)\left(\frac{1}{4}-m^{2}\right)^{2} .
\end{aligned}
$$

(vi) The contribution of particle hopping from site 5 to site 2 and coming back is similar to (v) and is given by Eq.(B4).

(vii) The particle hopping from site 6 to site 7 and returning back is also similar to $(\mathrm{v})$ and the contribution is again given by Eq. (B4).

(viii) The contribution of particle hopping from site 8 to site 7 and coming back is also similar to (v) and hence is given by Eq. (B4).

Adding all the contributions for case 1, we get the coefficient of $n_{1} n_{3}$ to be

$$
\frac{2 t^{2}}{\left(2 E_{p}+2 V_{p}\right)}\left[4\left(\frac{1}{2}-m\right)^{2}-4\left(\frac{1}{4}-m^{2}\right)\right] .
$$

Case 2: We consider contribution to NNN interaction when, among the three sites that are NN to the intermediate site, one of them is occupied and the other two are empty. Thus, compared to case 1 , there is an extra repulsion term $2 V_{p}$ in the denominator of the coefficient. Then, NNN interaction involves the following.

(i) The particle hops from site 1 to site 2 and comes back. Any one of the three neighboring sites of site 2, i.e., 3, 4, or 5 , is occupied; then, the contribution is:

$$
\begin{aligned}
& \approx-\frac{2 t^{2}}{\left(2 E_{p}+4 V_{p}\right)}\left[n_{1} n_{3}\left\langle 1-n_{4}\right\rangle\left\langle 1-n_{5}\right\rangle\right. \\
& \left.+n_{1}\left(1-n_{3}\right)\left\langle n_{4}\right\rangle\left\langle 1-n_{5}\right\rangle+n_{1}\left(1-n_{3}\right)\left\langle 1-n_{4}\right\rangle\left\langle n_{5}\right\rangle\right] \\
& \approx-\frac{2 t^{2}}{\left(2 E_{p}+4 V_{p}\right)}\left[n_{1} n_{3}\left(\frac{1}{2}-m\right)^{2}\right. \\
& \left.+2 n_{1}\left(1-n_{3}\right)\left(\frac{1}{4}-m^{2}\right)\right] .
\end{aligned}
$$

(ii) The particle hops from site 1 to site 7 and comes back. The situation is similar to (i) and hence the contribution is given by Eq. (B6). (iii) The particle hops from site 3 to site 2 and comes back. The resulting contribution is

$$
\begin{aligned}
& \approx-\frac{2 t^{2}}{\left(2 E_{p}+4 V_{p}\right)}\left[n_{3} n_{1}\left\langle 1-n_{4}\right\rangle\left\langle 1-n_{5}\right\rangle\right. \\
& \left.+n_{3}\left(1-n_{1}\right)\left\langle n_{5}\right\rangle\left\langle 1-n_{4}\right\rangle+n_{3}\left(1-n_{1}\right)\left\langle n_{4}\right\rangle\left\langle 1-n_{5}\right\rangle\right] \\
& \approx-\frac{2 t^{2}}{\left(2 E_{p}+4 V_{p}\right)}\left[n_{1} n_{3}\left(\frac{1}{2}-m\right)^{2}\right. \\
& \left.+2 n_{3}\left(1-n_{1}\right)\left(\frac{1}{4}-m^{2}\right)\right] .
\end{aligned}
$$

(iv) The particle hops from site 3 to site 7 and returns back. Since the situation is similar to (iii), the contribution is expressed by Eq. (B7).

(v) The particle hops from site 4 to site 2 and comes back. The contribution is

$$
\begin{aligned}
& \approx-\frac{2 t^{2}}{\left(2 E_{p}+4 V_{p}\right)}\left[\left\langle n_{4}\right\rangle\left(1-n_{1}\right) n_{3}\left\langle 1-n_{5}\right\rangle\right. \\
& \left.+\left\langle n_{4}\right\rangle n_{1}\left(1-n_{3}\right)\left\langle 1-n_{5}\right\rangle+\left\langle n_{4}\right\rangle\left(1-n_{1}\right)\left(1-n_{3}\right)\left\langle n_{5}\right\rangle\right] \\
& \approx-\frac{2 t^{2}}{\left(2 E_{p}+4 V_{p}\right)}\left[n_{1}\left(1-n_{3}\right)\left(\frac{1}{4}-m^{2}\right)\right. \\
& \left.+n_{3}\left(1-n_{1}\right)\left(\frac{1}{4}-m^{2}\right)+\left(1-n_{1}\right)\left(1-n_{3}\right)\left(\frac{1}{2}+m\right)^{2}\right] .
\end{aligned}
$$

(vi) The particle hops from site 5 to site 2 and comes back. The situation being similar to (v) leads to the contribution being given by Eq. (B8).

(vii) The particle hops from site 6 to site 7 and comes back; this circumstance is also similar to (v) and hence contribution same as in Eq. (B8).

(viii) The particle hops from site 8 to site 7 and comes back. Here too the contribution is given by Eq. (B8) since the circumstance is again similar to $(\mathrm{v})$.

Therefore, for case 2 , the sum total of the above contributions yields the coefficient of $n_{1} n_{3}$ to be

$$
\begin{aligned}
\frac{2 t^{2}}{\left(2 E_{p}+4 V_{p}\right)}\left[16\left(\frac{1}{4}-m^{2}\right)\right. & -4\left(\frac{1}{2}-m\right)^{2} \\
& \left.-4\left(\frac{1}{2}+m\right)^{2}\right] .
\end{aligned}
$$

Case 3: Contribution to NNN interaction when the intermediate site has any two of the three NN sites occupied with the other being empty. Then, compared to case 2 , the coefficient has an extra repulsion term $2 V_{p}$ in the denominator; consequently, NNN interaction involves the following.

(i) The particle hops from site 1 to site 2 and comes back; 
the resulting contribution is:

$$
\begin{aligned}
& \approx-\frac{2 t^{2}}{\left(2 E_{p}+6 V_{p}\right)}\left[n_{1} n_{3}\left\langle n_{4}\right\rangle\left\langle 1-n_{5}\right\rangle\right. \\
& \left.+n_{1} n_{3}\left\langle 1-n_{4}\right\rangle\left\langle n_{5}\right\rangle+n_{1}\left(1-n_{3}\right)\left\langle n_{4}\right\rangle\left\langle n_{5}\right\rangle\right] \\
& \approx-\frac{2 t^{2}}{\left(2 E_{p}+6 V_{p}\right)}[ \\
& {\left[2 n_{1} n_{3}\left(\frac{1}{4}-m^{2}\right)\right.} \\
& \left.+n_{1}\left(1-n_{3}\right)\left(\frac{1}{2}+m\right)^{2}\right] . \quad \text { (B1 }
\end{aligned}
$$

(ii) The particle hops from site 1 to site 7 and comes back. This situation is similar to (i) with the contribution being expressed by Eq. (B10).

(iii) The particle hops from site 3 to site 2 and returns; the ensuing contribution is:

$$
\begin{aligned}
& \approx-\frac{2 t^{2}}{\left(2 E_{p}+6 V_{p}\right)}\left[n_{3} n_{1}\left\langle 1-n_{4}\right\rangle\left\langle n_{5}\right\rangle\right. \\
& \left.+n_{3} n_{1}\left\langle n_{4}\right\rangle\left\langle 1-n_{5}\right\rangle+n_{3}\left(1-n_{1}\right)\left\langle n_{4}\right\rangle\left\langle n_{5}\right\rangle\right] \\
& \approx-\frac{2 t^{2}}{\left(2 E_{p}+6 V_{p}\right)}\left[\begin{array}{l}
2 n_{1} n_{3}\left(\frac{1}{4}-m^{2}\right) \\
\left.+n_{3}\left(1-n_{1}\right)\left(\frac{1}{2}+m\right)^{2}\right] \cdot \quad
\end{array}\right.
\end{aligned}
$$

(iv) The particle hops from site 3 to site 7 and comes back. The situation is similar to (iii) with the contribution being given by Eq. (B11).

(v) The particle hops from site 4 to site 2 and returns. This produces the contribution:

$$
\begin{aligned}
\approx & -\frac{2 t^{2}}{\left(2 E_{p}+6 V_{p}\right)}\left[\left\langle n_{4}\right\rangle n_{1} n_{3}\left\langle 1-n_{5}\right\rangle\right. \\
& \left.\quad+\left\langle n_{4}\right\rangle\left(1-n_{1}\right) n_{3}\left\langle n_{5}\right\rangle+\left\langle n_{4}\right\rangle n_{1}\left(1-n_{3}\right)\left\langle n_{5}\right\rangle\right] \\
\approx & -\frac{2 t^{2}}{\left(2 E_{p}+6 V_{p}\right)}\left[n_{1} n_{3}\left(\frac{1}{4}-m^{2}\right)\right. \\
& \left.+n_{1}\left(1-n_{3}\right)\left(\frac{1}{2}+m\right)^{2}+n_{3}\left(1-n_{1}\right)\left(\frac{1}{2}+m\right)^{2}\right] .
\end{aligned}
$$

(vi) The particle hops from site 5 to site 2 and comes back. The circumstance, being similar to (v), yields the contribution expressed in Eq. (B12).

(vii) The particle hops from site 6 to site 7 and comes back. The situation is also similar to (v) with the contribution being also given by Eq. (B12).

(viii) The particle hops from site 8 to site 7 and returns. Again the situation is similar to (v) with the contribution being again given by Eq. (B12).

Therefore, on adding all the various contributions for case
3 , we get the coefficient of $n_{1} n_{3}$ to be

$$
\frac{2 t^{2}}{\left(2 E_{p}+6 V_{p}\right)}\left[12\left(\frac{1}{2}+m\right)^{2}-12\left(\frac{1}{4}-m^{2}\right)\right] \text {. }
$$

Case 4: Contribution to NNN interaction when all of the three neighboring sites of the intermediate site are occupied. Here, compared to case 3, the coefficient has an extra repulsion term $2 V_{p}$ in the denominator. Then, NNN interaction involves the following.

(i) The particle hops from site 1 to site 2 and comes back. Consequently, the contribution is

$$
\begin{aligned}
& \approx-\frac{2 t^{2}}{\left(2 E_{p}+8 V_{p}\right)} n_{1} n_{3}\left\langle n_{4}\right\rangle\left\langle n_{5}\right\rangle \\
& \approx-\frac{2 t^{2}}{\left(2 E_{p}+8 V_{p}\right)} n_{1} n_{3}\left(\frac{1}{2}+m\right)^{2} .
\end{aligned}
$$

For all the following also the contribution is expressed by Eq. (B14) because the situation is similar to (i).

(ii) The particle hops from site 1 to site 7 and comes back.

(iii) The particle hops from site 3 to site 2 and returns.

(iv) The particle hops from site 3 to site 7 and comes back.

(v) The particle hops from site 4 to site 2 and comes back.

(vi) The particle hops from site 5 to site 2 and returns.

(vii) The particle hops from site 6 to site 7 and comes back.

(viii) The particle hops from site 8 to site 7 and returns. Therefore, for case 4 , the coefficient of $n_{1} n_{3}$ is given by

$$
-\frac{2 t^{2}}{\left(2 E_{p}+8 V_{p}\right)} \times 8\left(\frac{1}{2}+m\right)^{2} .
$$

Combining Eqs. (B5), (B9), (B13) and (B15), we finally get the coefficient of NNN repulsion (which acts along the diagonals) to be

$$
\begin{aligned}
V_{2}=2 t^{2} & {\left[\left(\frac{1}{2}-m\right)^{2} \frac{2 V_{p}}{\left(E_{p}+V_{p}\right)\left(E_{p}+2 V_{p}\right)}\right.} \\
& +\left(\frac{1}{4}-m^{2}\right) \frac{4 E_{p} V_{p}}{\left(E_{p}+V_{p}\right)\left(E_{p}+2 V_{p}\right)\left(E_{p}+3 V_{p}\right)} \\
& \left.+\left(\frac{1}{2}+m\right)^{2} \frac{2 E_{p} V_{p}}{\left(E_{p}+2 V_{p}\right)\left(E_{p}+3 V_{p}\right)\left(E_{p}+4 V_{p}\right)}\right] .
\end{aligned}
$$

To calculate the NNNN repulsion along the $\mathrm{x}$-axis ( $\mathrm{y}$ axis), we have to consider all the processes from which a term $n_{1} n_{4}\left(n_{1} n_{8}\right)$ can appear. Adding all those terms, we can see that the coefficient of NNNN repulsion is just half of the coefficient of NNN repulsion. The reason for this is that the relevant contributions are from only half of the eight situations considered in each of the above four occupancy cases (i.e, the four cases involving different number of occupied neighbors for the intermediate site). 


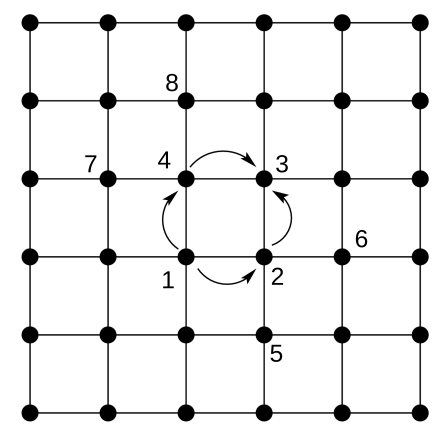

FIG. 12. Pictorial depiction of the process where a particle at site 1 hops to site 3 which is its NNN site along diagonal. The two possible paths for this process are indicated: hopping to site 3 via site 2 and site 4 .

\section{Appendix C: NNN hopping and NNNN hopping}

There are two possible hopping paths for a particle to arrive at a NNN site along the diagonals of the square lattice. For example, in Fig. 12 consider a particle hopping from site 1 to site 3 . It can either hop to site 2 first and then to site 3 or it can hop to site 4 followed by a hop to site 3 . Now, the coefficient of this process gets modified by the occupancy of the neighboring sites of the intermediate site. Without taking into account this effect, the process along any one path [on using Eq. A1] is given exactly by

$$
-\frac{t^{2} e^{-2\left(E_{p}+V_{p}\right) / \omega_{0}}}{\omega_{0}} G_{5}\left(2,2,1,1, \gamma^{2}\right) \sum_{<<i, j>>}\left(d_{i}^{\dagger} d_{j}+\text { H.c. }\right)
$$

where $\langle<i, j\rangle>$ denotes NNN pairs of sites along the diagonals. For large values of $g^{2}$, we have the following simplification for the coefficient in the above expression:

$$
\frac{t^{2} e^{-2\left(E_{p}+V_{p}\right) / \omega_{0}}}{\omega_{0}} G_{5}\left(2,2,1,1, \gamma^{2}\right) \approx \frac{t^{2} e^{-E_{p} / \omega_{0}}}{E_{p}+2 V_{p}} .
$$

Path 1: The particle hops from site 1 to site 3 via site 2. The coefficient of this process depends on the occupancy of the sites 5 and 6 which are the two neighboring sites of the intermediate site 2 .

Case 1: Contribution to NNN hopping when both the neighboring sites are empty:

$$
\begin{aligned}
& -\frac{t^{2} e^{-E_{p} / \omega_{0}}}{E_{p}+2 V_{p}} d_{3}^{\dagger} d_{1}\left(1-n_{5}\right)\left(1-n_{6}\right) \\
& \approx-\frac{t^{2} e^{-E_{p} / \omega_{0}}}{E_{p}+2 V_{p}} d_{3}^{\dagger} d_{1}\left\langle 1-n_{5}\right\rangle\left\langle 1-n_{6}\right\rangle \\
& \approx-\frac{t^{2} e^{-E_{p} / \omega_{0}}}{E_{p}+2 V_{p}}\left(\frac{1}{2}-m\right)^{2} d_{3}^{\dagger} d_{1} .
\end{aligned}
$$

Case 2: Contribution when any one of the neighboring sites is occupied (giving an extra repulsion $2 V_{p}$ in the denominator) and the other site is empty:

$$
\begin{aligned}
& \approx-\frac{t^{2} e^{-E_{p} / \omega_{0}}}{E_{p}+4 V_{p}} d_{3}^{\dagger} d_{1}\left[\left\langle n_{5}\right\rangle\left\langle 1-n_{6}\right\rangle+\left\langle 1-n_{5}\right\rangle\left\langle n_{6}\right\rangle\right] \\
& \approx-\frac{2 t^{2} e^{-E_{p} / \omega_{0}}}{E_{p}+4 V_{p}}\left(\frac{1}{4}-m^{2}\right) d_{3}^{\dagger} d_{1} .
\end{aligned}
$$

Case 3: Contribution when both the NN sites are occupied:

$$
\begin{aligned}
& \approx-\frac{t^{2} e^{-E_{p} / \omega_{0}}}{E_{p}+6 V_{p}} d_{3}^{\dagger} d_{1}\left\langle n_{5}\right\rangle\left\langle n_{6}\right\rangle \\
& \approx-\frac{t^{2} e^{-E_{p} / \omega_{0}}}{E_{p}+6 V_{p}}\left(\frac{1}{2}+m\right)^{2} d_{3}^{\dagger} d_{1} .
\end{aligned}
$$
be

Therefore, for path 1 , we get the coefficient of $d_{3}^{\dagger} d_{1}$ to

$$
\begin{aligned}
& -t^{2} e^{-E_{p} / \omega_{0}}\left[\left(\frac{1}{2}-m\right)^{2} \frac{1}{E_{p}+2 V_{p}}\right. \\
& \left.+\left(\frac{1}{4}-m^{2}\right) \frac{2}{E_{p}+4 V_{p}}+\left(\frac{1}{2}+m\right)^{2} \frac{1}{E_{p}+6 V_{p}}\right] .
\end{aligned}
$$

Path 2: The particle hops from site 1 to site 4 first and then to site 3 . The coefficient of this process gets modified depending on whether the sites 7 and 8 (NN to the intermediate site 4) are occupied or not.

Case 1 : Contribution when both the neighboring sites are empty. This situation is similar to case 1 of path 1 ; hence, the contribution is given by Eq. (C1).

Case 2 : Contribution when any one of the neighboring sites is occupied and the other one is empty. This is similar to case 2 of path 1 ; consequently, the contribution is expressed by Eq. (C2).

Case 3 : Contribution when both the NN sites are occupied. This circumstance is similar to case 3 of path 1; thus, the contribution is given by Eq. (C3).

Thus we see that path 2 yields the same coefficient [given by Eq. (C4)] for $d_{3}^{\dagger} d_{1}$ as path 1 . Combining the contributions from both the paths, a particle hopping to its NNN along diagonals can be expressed as $-t_{2} \sum_{<<i, j>>}\left(d_{i}^{\dagger} d_{j}+\right.$ H.c. $)$, where the coefficient $t_{2}$ is given by

$$
\begin{aligned}
t_{2} & =2 t^{2} e^{-E_{p} / \omega_{0}}\left[\left(\frac{1}{2}-m\right)^{2} \frac{1}{E_{p}+2 V_{p}}\right. \\
& \left.+\left(\frac{1}{4}-m^{2}\right) \frac{2}{E_{p}+4 V_{p}}+\left(\frac{1}{2}+m\right)^{2} \frac{1}{E_{p}+6 V_{p}}\right] .
\end{aligned}
$$

For the case of NNNN hopping (which occurs along the axes), there is only one possible path. Hence, the relevant coefficient $t_{3}$ for NNNN hopping is half of the coefficient for NNN hopping, i.e., $t_{3}=\frac{t_{2}}{2}$. 
1 M. Boninsegni, N. V. Prokof'ev, Rev. Modern Phys. 84, 759 (2012).

2 G. Bilbro, W. L. McMillan, Phys. Rev. B 14, 1887 (1976).

3 A. M. Gabovich, A. I. Voitenko and M. Ausloos, Phys. Rep. 367, 583 (2002).

${ }^{4}$ S. H. Blanton, R. T. Collins, K. H. Kelleher, L. D. Rotter, Z. Schlesinger, D. G. Hinks, and Y. Zheng, Phys. Rev. B 47, 996 (1993).

${ }^{5}$ R. L. Withers, J.A. Wilson, J. Phys. C 19, 4809 (1986).

6 J. Merino, R. H. McKenzie, Phys. Rev. Lett. 87, 237002 (2001)

7 W. W. Fuller, P. M. Chaikin, N.P. Ong, Phys. Rev. B 24, 1333 (1981)

8 A. Rusydi, W. Ku, B. Schulz, R. Rauer, I. Mahns, D. Qi, X. Gao, A.T.S. Wee, P. Abbamonte, H. Eisaki, Y. Fujimaki, S. Uchida, M. Rübhausen, Phys. Rev. Lett. 105, 026402 (2010).

9 P. Abbamonte, G. Blumberg, A. Rusydi, A. Gozar, P. G. Evans, T. Siegrist, L. Venema, H. Eisaki, E. D. Isaacs, G. A. Sawatzky, Nature 431, 1078 (2004).

10 A. Taraphder, R. Pandit, H. R. Krishnamurthy and T. V. Ramakrishnan, Int. J. Mod. Phys. B 10, 863 (1996).

11 For coexistence of superconductivity and antiferromagnetism in systems with strong electron-phonon interaction, see A. Y. Ganin, Y. Takabayashi, P. Jeglič, Denis Arčon, A. Potočnik, P. J. Baker, Y. Ohishi, M. T. McDonald, M. D. Tzirakis, A. McLennan, G. R. Darling, M. Takata, M. J. Rosseinsky, and K. Prassides, Nature 466, 221 (2010); M. Capone, M. Fabrizio, C. Castellani, and E. Tosatti, Rev. Mod. Phys. 81, 943 (2009); O. Gunnarsson, Rev. Mod. Phys. 69, 575 (1997).

12 C. M. Varma, Phys. Rev. Lett. 61, 2713 (1988).

13 R. Landig, L. Hruby, N. Dogra, M. Landini, R. Mottl, T. Donner, and T. Esslinger, Nature (London) 532, 476 (2016).

14 G. G. Batrouni and R. T. Scalettar, Phys. Rev. Lett. 84, 1599 (2000).

15 F. Hébert, G. G. Batrouni, R. T. Scalettar, G. Schmid, M. Troyer, and A. Dorneich, Phys. Rev. B 65, 014513 (2001).

16 Long Dang, Massimo Boninsegni and Lode Pollet, Phys. Rev. B 78, 132512 (2008).

17 B. Capogrosso-Sansone, C. Trefzger, M. Lewenstein, P. Zoller, and G. Pupillo Phys. Rev. Lett. 104, 125301 (2010).

18 Y.-C. Chen, R. G. Melko, S. Wessel, and Y.-J. Kao, Phys. Rev. B 77, 014524 (2008).

19 P. Sengupta, L. P. Pryadko, F. Alet, M. Troyer, and G. Schmid Phys. Rev. Lett. 94, 207202 (2005).

${ }^{20}$ G. Schmid and M. Troyer, Phys. Rev. Lett. 93, 067003 (2004).

${ }^{21}$ S. Kar and S. Yarlagadda, Ann. Phys. 375, 322 (2016); S. Datta, S. Yarlagadda, Solid State Commun. 150, 2040 (2010).

22 Xiao Huo, Yong-Yong Cui, Dali Wang, and Jian-Ping Lv, Phys. Rev. A 95, 023613 (2017).

23 S. Wessel and M. Troyer, Phys. Rev. Lett. 95, 127205 (2005).

24 D. Heidarian and K. Damle, Phys. Rev. Lett. 95, 127206 (2005).

25 A. Sen, P. Dutt, K. Damle, R. Moessner, Phys. Rev. Lett. 100, 147204 (2008)

${ }^{26}$ R. G. Melko, A. Paramekanti, A. A. Burkov, A. Vish- wanath, D. N. Sheng, and L. Balents, Phys. Rev. Lett. 95, 127207 (2005).

27 M. Boninsegni and N. Prokof'ev, Phys. Rev. Lett. 95, 237204 (2005).

28 R. G. Melko, A. Del Maestro, and A. A. Burkov, Phys. Rev. B 74, 214517 (2006).

29 L. Pollet, J. D. Picon, H. P. Büchler, and M. Troyer, Phys. Rev. Lett. 104, 125302 (2010).

30 S. Wessel, Phys. Rev. B 75, 174301 (2007).

31 J. Y. Gan, Y. C. Wen, J. Ye, T. Li, S.-J. Yang, and Y. Yu, Phys. Rev. B 75, 214509 (2007).

32 T. Mishra, R. V. Pai, and S. Mukerjee, Phys. Rev. A 89, 013615 (2014).

33 A. Ghosh and S. Yarlagadda, Phys. Rev. B 90, 045140 (2014).

34 T. Bilitewski and N. R. Cooper Phys. Rev. A 94, 023630 (2016).

35 For a review, see Holger Ulbrich and Markus Braden, Physica C 481, 31 (2012).

36 C. H. Chen, S.-W. Cheong, and A. S. Cooper, Phys. Rev. Lett. 71, 2461 (1993).

37 S.-W. Cheong, H. Y. Hwang, C. H. Chen, B. Batlogg, L. W. Rupp, Jr., and S. A. Carter Phys. Rev. B 49, 7088(R) (1994).

38 K. Ishizaka, Y. Taguchi, R. Kajimoto, H. Yoshizawa, and Y. Tokura, Phys. Rev. B 67, 184418 (2003).

39 R. Kajimoto, T. Kakeshita, H. Yoshizawa, T. Tanabe, T. Katsufuji, and Y. Tokura, Phys. Rev. B 64, 144432 (2001).

${ }^{40}$ H. Yoshizawa, T. Kakeshita, R. Kajimoto, T. Tanabe, T. Katsufuji, and Y. Tokura, Phys. Rev. B 61, R854(R) (2000).

41 P. G. Freeman, A. T. Boothroyd, D. Prabhakaran, M. Enderle, and C. Niedermayer, Phys. Rev. B 70, 024413 (2004).

42 L. Rademaker, Y. Pramudya, J. Zaanen, and V. Dobrosavljević, Phys. Rev. E 88, 032121 (2013).

43 T. Hotta and E. Dagotto, Phys. Rev. Lett. 92, 227201 (2004).

44 S. Yamamoto, T. Fujiwara, and Y. Hatsugai, Phys. Rev. B 76, 165114 (2007).

${ }^{45}$ K. Rościszewski and A. M. Oleś, J. Phys. Condens. Matter 23, 265601 (2011).

46 A. Ghosh and S. Yarlagadda (unpublished).

47 In one dimension, it has been shown in Ref. 33 that $t_{2}-V_{1}$ model yields a cSS. In 2D, it has been demonstrated in Ref. 21 that $t_{2}-t_{3}-V_{1}$ model generates a cSS; using similar logic, it can be shown that $t_{2}-V_{1}$ model also manifests cSS.

48 I. G. Lang and Yu. A. Firsov, Zh. Eksp. Teor. Fiz. 43, 1843 (1962) [Sov. Phys. JETP 16, 1301 (1963)].

49 R. Pankaj and S. Yarlagadda, Phys. Rev. B 86, 035453 (2012).

50 S. Reja, S. Yarlagadda, and P. B. Littlewood, Phys. Rev. B 84, 085127 (2011).

51 S. Reja, S. Yarlagadda, and P. B. Littlewood, Phys. Rev. B 86, 045110 (2012).

52 A. Dey, M. Q. Lone, and S. Yarlagadda, Phys. Rev. B 92, 094302 (2015).

53 A. W. Sandvik, AIP Conf. Proc. 1297, 135 (2010).

54 O. Nguyen and L. Dang, Eur. Phys. J. B 90, 71 (2017).

55 S. Wessel, Phys. Rev. B 86, 140501(R) (2012). 
${ }^{56}$ R. J. Cava, B. Batlogg, T. T. Palstra, J. J. Krajewski, W. F. Peck, Jr., A. P. Ramirez, and L. W. Rupp, Jr., Phys. Rev. B 43, 1229(R) (1991).

57 T. Katsufuji, T. Tanabe, T. Ishikawa, S. Yamanouchi, Y. Tokura, T. Kakeshita, R. Kajimoto, and H. Yoshizawa, Phys. Rev. B 60, R5097(R) (1999).

${ }^{58}$ I. A. Zaliznyak, J. P. Hill, J. M. Tranquada, R. Erwin, and Y. Moritomo, Phys. Rev. Lett. 85, 4353 (2000).

59 M. Cwik, M. Benomar, T. Finger, Y. Sidis, D. Senff, M. Reuther, T. Lorenz, and M. Braden, Phys. Rev. Lett. 102, 057201 (2009).

60 A. T. Boothroyd, P. Babkevich, D. Prabhakaran and P. G.
Freeman, Nature 471, 341 (2011).

61 Eric C. Andrade and Matthias Vojta, Phys. Rev. Lett. 109, 147201 (2012).

62 T. Lancaster, S. R. Giblin, G. Allodi, S. Bordignon, M. Mazzani, R. De Renzi, P. G. Freeman, P. J. Baker, F. L. Pratt, P. Babkevich, S. J. Blundell, A. T. Boothroyd, J. S. Möller, and D. Prabhakaran, Phys. Rev. B 89, 020405(R) (2014).

63 N. Hollmann, M. W. Haverkort, M. Cwik, M. Benomar, M. Reuther, A. Tanaka, and T. Lorenz, New J. Phys. 10, 023018 (2008). 